\title{
Mechanisms of Radiation-Induced Segregation in CrFeCoNi-based Single-Phase Concentrated Solid Solution Alloys
}

Mo-Rigen He, ${ }^{\mathrm{a},}{ }^{*}$ Shuai Wang, ${ }^{\mathrm{a}}$ Shi Shi, ${ }^{\mathrm{a}}$ Ke Jin, ${ }^{\mathrm{b}}$ Hongbin Bei, ${ }^{\mathrm{b}}$ Kazuhiro Yasuda, ${ }^{\mathrm{c}}$ Syo Matsumura, ${ }^{\mathrm{c}}$ Kenji Higashida, ${ }^{\mathrm{d}}$ and Ian M. Robertson ${ }^{\mathrm{a},{ }^{*}}$

${ }^{a}$ Department of Engineering Physics, University of Wisconsin-Madison, Madison, WI 53706, United States

${ }^{\mathrm{b}}$ Materials Science and Technology Division, Oak Ridge National Laboratory, Oak Ridge, TN 37831, United States

c Department of Applied Quantum Physics and Nuclear Engineering, Kyushu University, Fukuoka 819-0395, Japan

d Department of Materials Science and Engineering, Kyushu University, Fukuoka 819-0395, Japan

* Corresponding author.

E-mail address: rmo3@wisc.edu (M.R. He), irobertson@wisc.edu (I.M. Robertson) 


\begin{abstract}
Single-phase concentrated solid solution alloys have attracted wide interest due to their superior mechanical properties and enhanced radiation tolerance, which make them promising candidates for the structural applications in next-generation nuclear reactors. However, little has been understood about the intrinsic stability of their as-synthesized high-entropy configurations against radiation damage. Here we report the element segregation in $\mathrm{CrFeCoNi}, \mathrm{CrFeCoNiMn}$, and $\mathrm{CrFeCoNiPd}$ equiatomic alloys when subjected to $1250 \mathrm{kV}$ electron irradiations at $400{ }^{\circ} \mathrm{C}$ up to a damage level of 1 displacement per atom. $\mathrm{Cr} / \mathrm{Fe} / \mathrm{Mn} / \mathrm{Pd}$ can deplete and $\mathrm{Co} / \mathrm{Ni}$ can accumulate at radiation-induced dislocation loops, while the actively segregating elements are alloy-specific. Moreover, electron-irradiated matrix of $\mathrm{CrFeCoNiMn}$ and $\mathrm{CrFeCoNiPd}$ shows $\mathrm{L1}_{0}$ (NiMn)-type ordering decomposition and <001>-oriented spinodal decomposition between $\mathrm{Co} / \mathrm{Ni}$ and $\mathrm{Pd}$, respectively. These findings are rationalized based on the atomic size difference and enthalpy of mixing between the alloying elements, and identify a new important requirement to the design of radiation-tolerant alloys through modification of the composition.
\end{abstract}

Keywords:

Electron microscopy; Irradiated metals; Phase transformation; Segregation; Single-phase concentrated solid solution alloys. 


\section{Introduction}

The development of fusion and Generation-IV fission reactors demands advanced structural materials that can retain their performance under harsh operating environments, such as high temperature and high irradiation dose [1,2]. With respect to these challenges, single-phase concentrated solid solution alloys (SP-CSAs), including high-entropy alloys (HEAs) [3-5], have attracted increasing interest due to their high strength and ductility, improved fracture toughness, retarded energy dissipation, and suppressed damage accumulation [6-9]. These promising mechanical and physical properties rely on the extreme compositional complexity of this novel class of alloys, in which multiple principal elements (often in an equiatomic ratio) are randomly mixed in a simple crystal lattice. However, little is understood yet about the stability of such as-synthesized, high-entropy configurations under extreme environments, which are crucial to the reliable and predictable performance of these alloys in potential nuclear energy applications. The mechanisms of defect dynamics, radiation-induced segregation (RIS), and phase transition in multi-component CSAs are also of scientific significance, wherein the majority of knowledge are hitherto limited to binary and very few ternary systems $[10,11]$.

In particular, $\mathrm{CrFeCoNi}$-based equiatomic alloys have been extensively investigated as one of the few HEAs that can solidify as single-phase solid solution with a face-centered cubic (FCC) structure $[4,6,8,12]$. While long-range chemical ordering was confirmed to be absent after homogenization [13-15], the stability of such alloys under prolonged anneals [16,17] and electron/ion irradiations [18,19] still needs to be determined. For instance, a coarse-grained $\mathrm{CrFeCoNiMn}$ alloy was reported to decompose into three distinct phases: a body-centered cubic (BCC) $\mathrm{Cr}$-rich solid solution, a B2-type FeCo, and a $\mathrm{L} 1_{0}$-type NiMn, after annealing at $500{ }^{\circ} \mathrm{C}$ for 500 days, but remained stable after annealing at $900{ }^{\circ} \mathrm{C}$ for 500 days [17]. Similar decomposition occurred in a nanocrystalline (with grain sizes of $\sim 50 \mathrm{~nm}$ ) $\mathrm{CrFeCoNiMn}$ alloy produced by high-pressure torsion, but after annealing at $450{ }^{\circ} \mathrm{C}$ for 15 hours [16]. On the other

hand, a nanocrystalline (with grain sizes of $\sim 10 \mathrm{~nm}$ ) $\mathrm{CrFeCoNiCu}$ alloy was reported to retain its primary phase, i.e., FCC solid solution, when irradiated by $2 \mathrm{MeV}$ electrons at $25^{\circ} \mathrm{C}$ and $500{ }^{\circ} \mathrm{C}$ 
up to a damage level of 40 displacements per atom (dpa), although BCC solid solution, B2-type $\mathrm{FeCo}$, and $\mathrm{L1}_{0}$-type $\mathrm{NiFe}$ phases were formed earlier [18]. No phase change was noticed in a coarse-grained CrMnFeNi alloy irradiated by $5.8 \mathrm{MeV} \mathrm{Ni}^{+}$ions at $400 \sim 700{ }^{\circ} \mathrm{C}$ up to $10 \mathrm{dpa}$, while solute segregation was observed at grain boundaries [19]. Grain boundaries not only serve as sinks for the annihilation of radiation-induced defects [20], but also short paths for the biased diffusion of alloying elements [19,21], thus, they may be either beneficial or detrimental to the stability of SP-CSAs. In this regard, here we focus on the intrinsic segregation of single-crystalline or coarse-grained, equiatomic $\mathrm{CrFeCoNi}, \mathrm{CrFeCoNiMn}$, and $\mathrm{CrFeCoNiPd}$ alloys under high-voltage electron irradiations in which point defects but not displacement cascades are generated. The enrichment and depletion of alloying elements at radiation-induced dislocation loops as well as the embryos of phase decomposition within irradiated matrix are quantified using a scanning/transmission electron microscope (STEM). The factors that govern the nature and mechanisms of RIS in SP-CSAs are discussed.

\section{Experimental}

The three alloys studied in this work were synthesized using high-purity $(>99.9 \%)$ elemental metals by arc melting and drop casting into copper molds. Single-crystalline CrFeCoNi was then obtained by directional solidification in a floating zone furnace under an Ar gas atmosphere [22], while $\mathrm{CrFeCoNiMn}$ and $\mathrm{CrFeCoNiPd}$ with very coarse grains over $500 \mu \mathrm{m}$ were obtained by homogenization at $1200{ }^{\circ} \mathrm{C}$ for 1 week [23]. Disks with a diameter of $3 \mathrm{~mm}$ were thinned to electron-transparency by twin-jet electropolishing using an electrolyte of $10 \%$ perchloric acid + $90 \%$ ethanol at a temperature of $-30 \sim-20^{\circ} \mathrm{C}$ and a voltage of $14 \sim 16 \mathrm{~V}$. Irradiations by $1250 \mathrm{kV}$ electrons were carried out inside a JEOL JEM-1300NEF high-voltage electron microscope at a nominal temperature of $400{ }^{\circ} \mathrm{C}$. The steady temperature increase due to electron irradiation was estimated to be within $30{ }^{\circ} \mathrm{C}$ (see Appendix A for details). A damage level of $1 \mathrm{dpa}$ was obtained by approximately 30 minutes of irradiation with an electron dose rate of $\sim 5 \times 10^{18} \mathrm{e} \cdot \mathrm{cm}^{-2} \cdot \mathrm{s}^{-1}$. All irradiations were conducted in areas with a normal close to [001] zone axis (ZA). Post-mortem 
characterization of dislocation loops was performed using a FEI Tecnai TF-30 TEM operating at $300 \mathrm{kV}$. The Burgers vector (b), habit plane, and nature of each loop was identified using the $\mathbf{g} \cdot \mathbf{b}$ invisibility criterion and the inside-outside contrast method under variant (g, 3.1g) weak-beam dark-field (WBDF) conditions, as described in our previous work [24]. Compositional profiles at dislocation loops and in the irradiated matrix were measured by energy dispersive spectroscopy (EDS) attached to a FEI TITAN STEM operating at $200 \mathrm{kV}$.

\section{Results and Discussions}

\subsection{Structure of dislocation loops}

The structure of defects in $\mathrm{CrFeCoNi}$ as induced by $1250 \mathrm{kV}$ electron irradiation at $400{ }^{\circ} \mathrm{C}$ and their growth behavior up to $1 \mathrm{dpa}$ has been described previously [24]. As represented in Fig. 1(a), two distinct types of defects, i.e., elliptical Frank loops with $\mathbf{b}=\langle 111\rangle / 3$ and rhombus perfect loops with $\mathbf{b}=\langle 110>/ 2$, were identified under WBDF imaging conditions. All dislocation loops were of interstitial-type [24], with the (apparent) habit plane perpendicular to b. While edge-on view of Frank loop (Fig. 2(a)) shows the flat $\{111\}$ habit plane, perfect loop (Fig. 2(c)) might possess non-planar configuration composed of alternating $\{111\}$ planes, since the $\{110\}$ habit plane is not close-packed in FCC lattice. This can be implied by the formation of perfect loops, with similar crystallographic characters but of vacancy-type, in quenched Al-based alloys [25] via the dissociation and reorientation of Frank loops [26]. We also note that Fig. 2(a) and 2(c) (and all STEM images hereinafter) were recorded in annular dark-field (ADF) mode, in which the contrast is sensitive to atomic mass $(\mathrm{Z})$ but is also contributed by the scattering of lattice defects.

Fig. 1(b) and 1(c) show the representative defect structure in CrFeCoNiMn and CrFeCoNiPd, respectively, which were irradiated at the same conditions as of $\mathrm{CrFeCoNi}(1250 \mathrm{kV}$ electrons, $400{ }^{\circ} \mathrm{C}, 1 \mathrm{dpa}$ ). Still, all dislocation loops were of interstitial-type. Perfect loops were present at a lower fraction in these quinary alloys, possibly due to the reduction in stacking fault energy [27]. The number density of loops was much lower, although the loop sizes were larger, in 
$\mathrm{CrFeCoNiMn}$ and $\mathrm{CrFeCoNiPd}$ as compared to $\mathrm{CrFeCoNi}$. While no evidence has been found for the difference in the displacement threshold energy of these complex alloys, the lower density of loop nucleus can be attributed to the enhanced recombination of point defects associated with the oversized elements (Mn or Pd) [28]. A detailed quantitative analysis of loop growth behavior will be presented elsewhere, and the following section (3.2) will focus on the nature of RIS at loop habit planes. In addition, one may notice the more "noisy" background in Fig. 1(b) and 1(c), which will be related in later sections (3.3 and 3.4) to the early stage of phase decomposition in the electron-irradiated matrix of $\mathrm{CrFeCoNiMn}$ and $\mathrm{CrFeCoNiPd}$, respectively.

\subsection{RIS at dislocation loops}

Fig. 2(b) and 2(d) show the compositional profiles across the habit plane of a Frank loop and a perfect loop (with major axis lengths of 70 100 nm) in $\mathrm{CrFeCoNi}$, respectively. Both types of loops showed a depletion of $\mathrm{Cr}$ and Fe along with an enrichment of $\mathrm{Co}$ and $\mathrm{Ni}$ to a comparable level of $2 \sim 3$ at. \% relative to the composition of irradiated matrix, which was identical to that of un-irradiated matrix. Segregation was observed within a wider range across the perfect loop, probably due to its atomically wavy configuration. RIS at defect sinks (e.g., dislocation loops and grain boundaries) has been modeled based on the fact that the migration of interstitials and vacancies in alloys can be chemically inhomogeneous [11]. While direct assessment of atomic diffusion in CrFeCoNi-based alloys has not been reported, Fig. 2(b) and 2(d) accord with recent molecular dynamics simulations of $\mathrm{Ni}_{0.5} \mathrm{Fe}_{0.5}$ and $\mathrm{Ni}_{0.8} \mathrm{Cr}_{0.2}$ concentrated $\mathrm{FCC}$ alloys [29,30], which showed that the interstitial diffusion was predominantly contributed by $\mathrm{Ni}$, thus leading to the enrichment of $\mathrm{Ni}$ at the interstitial-type Frank loops formed by the accumulation of point defects. RIS behavior of $\mathrm{CrFeCoNi}$ also agrees well with the interstitial-binding mechanism as in binary alloys [31], which is governed by the atomic size difference $(\Delta a)$ between the alloying

elements. Thereby, the larger $\mathrm{Cr} / \mathrm{Fe}$ atoms were not favored, while the smaller $\mathrm{Co} / \mathrm{Ni}$ atoms were favored at the interstitial-type loops. The relatively small $\Delta a$ in $\mathrm{CrFeCoNi}$ (see Table 1 [32]) was also responsible for the weak RIS as observed. 
We further demonstrate that RIS in CSAs can be more complicated and dependent on the species of alloying elements. Fig. 3(a) and 3(c) show edge-on view of a Frank loop (with major axis lengths of 120 150 nm) in CrFeCoNiMn and CrFeCoNiPd, respectively, and Fig. 3(b) and 3(d) show the compositional profiles across the habit planes. Intriguingly, while a depletion of $\mathrm{Mn} / \mathrm{Pd}$ and an enrichment of Co was observed to a significant level of 5 7 at. \% relative to the un-irradiated matrix, the depletion of $\mathrm{Cr} / \mathrm{Fe}$ and the enrichment of $\mathrm{Ni}$ was much weaker in $\mathrm{CrFeCoNiPd}$, and was even unresolvable (i.e., below error bars) in CrFeCoNiMn. Although the depletion of $\mathrm{Mn} / \mathrm{Pd}$ at interstitial-type loops can still be rationalized by their large atomic sizes (see Table 1 [32]), the distinct enrichment of $\mathrm{Co}$ and $\mathrm{Ni}$ indicated that RIS was not solely governed by $\Delta a$. The enthalpy of mixing $\left(\Delta H_{\text {mix }}\right)$ between all relevant elements, as calculated in equiatomic binary alloys $[23,32,33]$, are further listed in Table 1 . We notice that Ni-Mn possess a very negative $\Delta H_{\text {mix }}$ corresponding to a strong tendency to coexist, while their large $\Delta a$ conversely requires a segregation at dislocation loops. This competition might eventually neutralize the variation of $\mathrm{Ni}$ concentration. Along this line, the significant segregation between Co-Mn could be attributed to their large $\Delta a$ overcoming a less negative $\Delta H_{\text {mix }}$. For CrFeCoNiPd, the large $\Delta a$ between $\mathrm{Co} / \mathrm{Ni}$ and $\mathrm{Pd}$ coincides with the positive (or near-zero) $\Delta H_{\text {mix }}$, which favors their segregation at dislocation loops. Even so, the weaker segregation between Ni-Pd than Co-Pd was contrary to the more positive $\Delta H_{\text {mix }}$. The weak depletion of $\mathrm{Cr}$ was also not well reasoned in view of the very negative $\Delta H_{\text {mix }}$ between $\mathrm{Cr}-\mathrm{Pd}$. Overall, further experimental and theoretical efforts are required for the quantification of RIS behaviors in multi-component CSAs, which could not be simply assessed by the combinations of their binary/ternary subsystems.

\subsection{RIS in irradiated matrix of $\mathrm{CrFeCoNiMn}$}

In the remainder of this paper, RIS in the electron-irradiated matrix of SP-CSAs provides a unique probe to the configurational energy landscape of these complex alloys, since the embryos of phase transition (if any) are more likely to survive in the absence of displacement cascades and away from external defect sinks. To start with, CrFeCoNi did not present any observable 
phase change up to $1 \mathrm{dpa}$ (see Appendix B for details). In contrast, the irradiated matrix of CrFeCoNiMn showed grainy contrast in the Z-sensitive ADF-STEM image (Fig. 4(a)), and the corresponding selected area diffraction pattern (SADP) showed weak but certainly visible $\{100\}$ and $\{110\}$ families of reflections (Fig. 4(b)) that are forbidden for a disordered FCC lattice, indicating the emergence of long-range chemical ordering as induced by electron irradiation. In comparison, the un-irradiated matrix, which was away from the illumination of high-voltage electrons but was also annealed along with the irradiated matrix (the difference in temperature due to electron irradiation was insignificant, see Appendix A), merely showed slight oxidation on the surface of the TEM foil (marked by arrows in Fig. 4(c), the lattice-like contrast came from Moiré interference between FCC matrix and the spinel-type oxides, see Fig. 4(d)).

To assess the chemical nature of ordering, we further quantified the degree of covariance (DOC) between each pair of elements (A-B) in CrFeCoNiMn, which is defined as:

$$
D O C=r_{\mathrm{A}-\mathrm{B}} \frac{\sigma_{\mathrm{A}}}{\mu_{\mathrm{A}}} \frac{\sigma_{\mathrm{B}}}{\mu_{\mathrm{B}}} .
$$

Practically, composition of a specific region (i.e., irradiated or un-irradiated matrix) was measured by EDS at multiple random locations. Hence, $\mu_{\mathrm{A}}\left(\mu_{\mathrm{B}}\right)$ and $\sigma_{\mathrm{A}}\left(\sigma_{\mathrm{B}}\right)$ are the mean value and standard deviation of the atomic concentration of element $\mathrm{A}(\mathrm{B})$, respectively, and $r_{\mathrm{A}-\mathrm{B}}$ is the Pearson correlation coefficient between the two elements. As seen in Fig. 5(a) and 5(b), standard deviations of all elements were increased due to electron irradiation. However, only $\mathrm{Cr}-\mathrm{Fe}$ and Ni-Mn show a dramatically augmented and positive DOC, indicating spatial separation between these two pairs of tightly coupled elements (Co was not actively involved in any coupling). The covariance of Ni-Mn can be attributed to their very negative $\Delta H_{\text {mix }}[23,32]$ (see Table 1 ), and accorded well with previous reports that $\mathrm{CrFeCoNiMn}$ was decomposed by annealing at intermediate temperatures, generating a $\mathrm{L1}_{0}$-type ordered NiMn phase $[14,17]$. In the meantime, the $\{100\}$ and $\{110\}$ extra spots in Fig. 4(b), with the intensity $(I)$ showing $I_{\{100\}}>I_{\{110\}}>0$, can only be explained by a mixture of $\mathrm{L} 1_{0}$-type ordered domains with the three different orientations (see Fig. 4(e)), although it is still unclear why ordering along [001] orientation, which was close 
to the normal of TEM thin foil, was not favored. Other major types of ordered structures, such as $\mathrm{L1}_{2}$, B2, and $\sigma$-phase, can be further excluded by examining their SADPs in [001] ZA (see Fig. 4(f-h)), as calculated based on kinematical approximation [34]. For instance, $\mathrm{L}_{2}$ phase yields $I_{\{100\}} \sim I_{\{110\}}$, B2 phase yields $I_{\{100\}}<I_{\{110\}}$, and each $L 1_{0}$ domain only provides a part of extra spots. Overall, we conclude that high-voltage electron irradiation induced a $\mathrm{L}_{0}$ (NiMn)-type ordering decomposition in $\mathrm{CrFeCoNiMn}$, which supports the conjecture that SP-CSA (i.e., the high-entropy configuration) may not always be the energetically stable state, especially at intermediate temperatures and under irradiations, and that $\Delta H_{\text {mix }}$ may play a greater role in governing the phase stability of multi-component alloys [23].

\subsection{RIS in irradiated matrix of $\mathrm{CrFeCoNiPd}$}

The electron-irradiated matrix of $\mathrm{CrFeCoNiPd}$ also manifested intriguing decomposition behavior. Unlike the spatially dispersed ordering in $\mathrm{CrFeCoNiMn}$, a self-organized, tweed-like microstructure with modulated contrast along [100] and [010] directions was noticed in ADF-STEM image (Fig. 6(a)), and streaks along the same directions were observed in all diffraction spots in [001] ZA (Fig. 6(b)). Moreover, transverse splits along [101] direction were observed for the spots with larger $|\mathbf{g}|$ (i.e., the length of reciprocal vector) in [101] ZA, while those with smaller $|\mathbf{g}|$ still showed longitudinal streaks along [010] direction (Fig. 6(c)). These features indicate that the structural modulations, and consequently the streaks of all reciprocal sites, were along all six $<001>$ directions in three-dimensional reciprocal space, as illustrated in Fig. 6(g). In comparison, the un-irradiated matrix did not present any directional variation of contrast (Fig. 6(d)), nor diffuse intensity in SADPs (Fig. 6(e) and 6(f)).

The tweed-like microstructure was further associated with a segregation between $\mathrm{Co} / \mathrm{Ni}$ and Pd. As demonstrated in Fig. 7(a) and 7(b), the regions rich of $\mathrm{Co} / \mathrm{Ni}$ and $\mathrm{Pd}$ correlated well with darker and brighter contrast in the Z-sensitive ADF-STEM image, respectively. $\mathrm{Cr} / \mathrm{Fe}$ were not significantly modulated compared to the un-irradiated matrix. Assuming Vegard's law [35], the variation of lattice parameter due to the segregation between $\mathrm{Co} / \mathrm{Ni}$ and $\mathrm{Pd}$ was no larger than \pm 
$0.3 \%$. Nonetheless, the <001>-oriented streaks in SADPs, which were up to $\pm 3 \sim 5 \%$ of $\left|\mathbf{g}_{200}\right|$, can be modeled by a quasi-periodic modulation, e.g., spinodal decomposition, to the atomic scattering factor. This is herein simplified as a one-dimensional sinusoidal wave, $A \cdot \sin (2 \pi x / \lambda)$, with a wavelength $\lambda=5 \mathrm{~nm}$ and a relative amplitude $A=0.2$, as indicated by Fig. 7(a) and 7(b), respectively. Diffraction profile along each [001] direction can be thus calculated based on the following Fourier transform [36]:

$$
I(g)=\left|\boldsymbol{F}\left\{U_{0}\left(\frac{x}{a}\right) \cdot\left[1+A \cdot \sin \left(\frac{2 \pi x}{\lambda}\right)\right]\right\}\right|^{2}=I(g)+A^{2} \cdot\left[I \quad\left(g-\frac{1}{\lambda}\right)+I\left(g+\frac{1}{\lambda}\right)\right] .
$$

Here, $U_{0}(x / a)$ and $I_{0}(g)$ is the atomic potential and diffraction of FCC lattice without modulation. As shown in Fig. 7(c), the diffuse intensity on the shoulder of experimental $\{220\}$ peak was exposed by deconvolution as a pair of extra peaks centering at $\sim 0.2 \mathrm{~nm}^{-1}$, according well with the calculated diffraction of spinodal decomposition.

Tweed-like microstructure and streaks in SADPs were recognized as the signature of shear-coupled, pre-martensitic transformation in various FCC- and BCC-based ordered alloys [37-39], however, all along <110> directions. For instance, $<110>$-oriented tweeds appeared in a $\mathrm{Fe}_{0.506} \mathrm{Pd}_{0.494}$ alloy in the initial state of ordering transformation from $\mathrm{FCC}$ to $\mathrm{L1}_{0}$ structure [37]. By contrast, spinodal decomposition in cubic crystals with an elastic anisotropic ratio $A=$ $2 C_{44} /\left(C_{11}-C_{12}\right)>1$, such as $\mathrm{Ni}$ and $\mathrm{Pd}[40]$, was known to self-organize along the soft $<001>$ directions [41]. Although spinodal decomposition was not reported in Co-Pd and Ni-Pd phase diagrams at $400{ }^{\circ} \mathrm{C}[42]$, multi-component CSAs might possess $\Delta H_{\text {mix }}$ values that are different from their binary subsystems. Moreover, X-ray diffuse scattering measurements indicated that equiatomic Ni-Pd solid solution, which was homogenized at $1100{ }^{\circ} \mathrm{C}$ for 1 week, still contained $<001>$-oriented segregating clusters with lengths of 2 5 unit cells [43], reflecting the role of a positive $\Delta H_{\text {mix }}$ [33]. These might serve as the nucleus for spinodal decomposition, which was further prompted by the point defect flows generated by electron irradiation [44], and finally evolved into the quasi-periodic microstructure seen in Fig. 6(a). 


\section{Discussions}

4.1. Governing mechanisms of RIS and the effect of damage type

Our comprehensive S/TEM characterization of electron-irradiated $\mathrm{CrFeCoNi}, \mathrm{CrFeCoNiMn}$, and $\mathrm{CrFeCoNiPd}$ alloys provides unique understanding of the mechanisms of RIS both at the defect sinks and within the intrinsic matrix. While various criteria have been proposed for the stabilization of single-phase solid solution in HEAs based on $\Delta a$ and $\Delta H_{\text {mix }}$ between the alloying elements $[45,46]$, we have shown above that the nature of RIS can be rationalized by the same sets of parameters, at least on a qualitative level. First, $\Delta a$ plays a dominant role in determining the enrichment vs. depletion of alloying elements at the dislocation loops associated with a local stress field, with the only exception of $\mathrm{Ni}$ in $\mathrm{CrFeCoNiMn}$, when the thermodynamic binding between Ni-Mn is very strong (we note that the segregation between Co-Mn is still determined by their large $\Delta a$ ). Meanwhile, phase instability of the electron-irradiated matrix can be triggered by $\Delta H_{\text {mix }}$ of a specific element pair, even if single-phase solid solution is still favored by the overall mixing enthalpy $\Omega=\sum_{i \neq j} 4 \Delta H_{\text {mix }}^{i-j} c_{i} c_{j}$, e.g., when $-12 \mathrm{~kJ} / \mathrm{mol} \leq \Omega \leq 3 \mathrm{~kJ} / \mathrm{mol}$, in the as-synthesized state [46]. For instance, the ordering decomposition in $\mathrm{CrFeCoNiMn}$ is driven by the very negative $\Delta H_{\text {mix }}$ between $\mathrm{Ni}-\mathrm{Mn}$, and the spinodal decomposition in $\mathrm{CrFeCoNiPd}$ is driven by the positive $\Delta H_{\text {mix }}$ between $\mathrm{Co} / \mathrm{Ni}$ and $\mathrm{Pd}$. By contrast, $\mathrm{CrFeCoNi}$, with all element pairs satisfying $-7 \mathrm{~kJ} / \mathrm{mol} \leq \Delta H_{\mathrm{mix}} \leq 0$, remains stable up to $1 \mathrm{dpa}$. Therefore, a new and stricter criterion may exist for the stabilization of SP-CSAs under irradiation environments.

It is also valuable to discuss the nature and mechanisms of RIS under the damage of different types of particles, e.g., electrons, protons, heavy ions, and neutrons. Grain boundaries of several austenitic stainless steels presented RIS with identical features, i.e., enrichment of Ni and depletion of $\mathrm{Cr}$, and comparable magnitude under varied irradiation conditions [28,47-49]. However, there have not been sufficient experimental results on the RIS of HEAs, regardless of damage type. A recent study of $\mathrm{Cr}_{18} \mathrm{Mn}_{27} \mathrm{Fe}_{27} \mathrm{Ni}_{28}$, a non-equiatomic SP-CSA with a FCC structure [19], showed an enrichment of $\mathrm{Ni}$ by $\sim 5$ at. \% along with a depletion of Mn by $\sim 5$ at. \% and $\mathrm{Cr} / \mathrm{Fe}$ by $\sim 2$ at. $\%$ at grain boundaries when irradiated by $5.8 \mathrm{MeV} \mathrm{Ni}^{+}$ions at $500{ }^{\circ} \mathrm{C}$ up to 
10 dpa. These features can be well explained by the interstitial-binding mechanism, as governed by $\Delta a$ (see Table 1), or the inverse Kirkendall effect [48], since grain boundary is also the sink of vacancy diffusion. However, it seems that RIS was not significantly influenced by the strong binding between Ni-Mn, and no phase change was noticed in the ion-irradiated matrix [19]. While RIS behaviors may depend on the species and concentrations of alloying elements, we propose that irradiations by heavy ions, which generate far-from-equilibrium states (i.e., displacement cascades) on a much larger spatial and temporal scale compared to electrons [50], may suppress the role of thermodynamic parameters, such as $\Delta H_{\text {mix }}$. In consequence, the nature and magnitude of RIS may be primarily governed by the solute-coupled migration of point defects and clusters, which is further modulated by the concentrated alloying of SP-CSAs [51].

\subsection{Resistance to RIS and the role of compositional complexity}

We finally emphasize the role of compositional complexity in RIS-resistance, which is evaluated based on the relative magnitude of element enrichment and depletion at defect sinks (e.g., dislocation loops, grain boundaries, and free surfaces). Fig. 8(a) compares the electron-irradiated $\mathrm{CrFeCoNi}$ alloy in this study with common austenitic stainless steels $[48,49,52-54]$ and several Ni-based dilute and concentrated binary alloys $[55,56]$, which were irradiated under varied conditions and up to a similar damage level. The evolution of RIS as a function of temperature and damage level will be studied in future. Although the effectiveness of defect sinks may depend on their character (indeed, dislocation loops and grain boundaries showed comparable RIS behaviors [54]), CrFeCoNi unambiguously manifests an improved RIS-resistance, which can be attributed to the reduced mobility [30] and enhanced recombination [28] of point defects. This alloy also shows promising phase stability under irradiation, and our results of $\mathrm{CrFeCoNiMn}$ and $\mathrm{CrFeCoNiPd}$ (also see Fig. 8(b)) indicate that RIS-resistance may not always be monotonically improved by increasing the number of alloying elements. As a closing remark, radiation-induced structural modulation may impose an additional energy barrier to point defect migration, and further contribute to the radiation tolerance and physical (i.e., 
transport) properties of CSAs and HEAs [8,27]. The consequence of RIS to the comprehensive performance of these complex alloys should also be the focus of future studies.

\section{Conclusions}

In summary, mechanisms of RIS in $\mathrm{CrFeCoNi}, \mathrm{CrFeCoNiMn}$, and $\mathrm{CrFeCoNiPd}$ equiatomic alloys are experimentally revealed, to our knowledge, for the first time. $\mathrm{Cr} / \mathrm{Fe} / \mathrm{Mn} / \mathrm{Pd}$ can deplete and $\mathrm{Co} / \mathrm{Ni}$ can accumulate at radiation-induced dislocation loops, while the actively segregating elements are alloy-specific and primarily determined by the atomic size difference. While the electron-irradiated matrix of $\mathrm{CrFeCoNi}$ shows promising phase stability, $\mathrm{CrFeCoNiMn}$ and CrFeCoNiPd show $\mathrm{L}_{0}$ (NiMn)-type ordering decomposition and <001>-oriented spinodal decomposition between $\mathrm{Co} / \mathrm{Ni}$ and $\mathrm{Pd}$, respectively, as governed by the enthalpy of mixing. Nonetheless, a quantitative interpretation and prediction of RIS behaviors in multi-component CSAs based on the knowledge of their binary/ternary subsystems is still a great challenge. RIS-resistance of CSAs and HEAs is inherent to their compositional complexity, but also dependent on the species of alloying elements. Overall, our findings identify a new important requirement to the design of radiation-tolerant alloys through modification of the composition.

\section{Appendix A. Steady temperature increase due to electron irradiation}

The TEM thin foil under electron irradiation is approximated as shown in Fig. A. Electron beam with a radius $R_{\mathrm{e}}$ and an intensity $J_{\mathrm{e}}$ is uniformly irradiated onto and completely transmitted through the central area of the specimen with a uniform thickness. The thickness out of irradiated area gradually increases with the distance $(r)$ to center (we note that the value of thickness is not needed in the following calculations). Heat flow is restricted in radial direction of the specimen with a thermal conductivity $\kappa$ (i.e., assuming no heat dissipation into the vacuum), and the temperature $(T)$ at outer radius $\left(R_{0}\right)$ of specimen is the nominal temperature of irradiation experiments $\left(T_{0}\right)$. The steady-state heat transfer equation in cylindrical coordinates is written as: 
$\kappa\left(\frac{d^{2} T}{d r^{2}}+\frac{1}{r} \frac{d T}{d r}\right)+J_{\mathrm{H}}=0$, when $0 \leq r \leq R_{\mathrm{e}} ;$

$\kappa\left(\frac{d^{2} T}{d r^{2}}+\frac{1}{r} \frac{d T}{d r}\right)=0$, when $R_{\mathrm{e}} \leq r \leq R_{0}$

Here, $J_{\mathrm{H}}$ is the input power density determined as:

$J_{\mathrm{H}}=\frac{\rho Q J_{\mathrm{e}}}{e}$, where $\rho$ is the density of specimen, and $Q$ is the total stopping power for electrons. The solution of these equations is (see Fig. A):

$$
\begin{aligned}
& T(r)=T_{\max }-\frac{J_{\mathrm{H}}}{4 \kappa} r^{2}, \text { when } 0 \leq r \leq R_{\mathrm{e}} ; \\
& T(r)=T_{0}-\frac{J_{\mathrm{H}}}{2 \kappa} R_{\mathrm{e}}^{2} \ln \frac{r}{R_{0}}, \text { when } R_{\mathrm{e}} \leq r \leq R_{0} .
\end{aligned}
$$

In the end, the maximum temperature increase is determined by:

$$
T_{\max }-T_{0}=\frac{\rho Q J_{\mathrm{e}} R_{\mathrm{e}}^{2}}{4 \kappa e}\left(1+2 \ln \frac{R_{0}}{R_{\mathrm{e}}}\right) .
$$

For our experiments, $J_{\mathrm{e}}=5 \times 10^{18} \mathrm{e} \cdot \mathrm{cm}^{-2} \cdot \mathrm{s}^{-1}, R_{\mathrm{e}}=3 \mu \mathrm{m}$, and $R_{0}=1.5 \mathrm{~mm}$. The density and stopping power of $\mathrm{CrFeCoNi-based} \mathrm{alloys} \mathrm{are} \mathrm{approximated} \mathrm{as} \mathrm{that} \mathrm{of} \mathrm{pure} \mathrm{Ni,} \mathrm{i.e.,} \rho=8.91$ $\mathrm{g} \cdot \mathrm{cm}^{-3}$, and $Q=1.37 \mathrm{MeV} \cdot \mathrm{cm}^{2} \cdot \mathrm{g}^{-1}$ for $1250 \mathrm{kV}$ electrons [57]. However, thermal conductivity of these CSAs was known to be significantly suppressed than that of pure Ni. For instance, $\kappa=$ $(10.3 \sim 13.7) \mathrm{W} \cdot \mathrm{m}^{-1} \cdot \mathrm{K}^{-1}$ at $300 \mathrm{~K}[58]$. These data yield a steady temperature increase of no larger than $30 \mathrm{~K}$, which should not be significant since $T_{0}=673 \mathrm{~K}$.

\section{Appendix B. Electron-irradiated matrix of $\mathrm{CrFeCoNi}$}

Structural changes were not observed in the electron-irradiated matrix of CrFeCoNi (up to 1 dpa), as confirmed by the featureless ADF-STEM image (Fig. B(a)) and SADP (Fig. B(b)). The slight oxidation can be indexed as corundum-type oxides (such as $\mathrm{Cr}_{2} \mathrm{O}_{3}$ ). Nonetheless, the DOC was consistently negligible, even after irradiation, for all element pairs (Fig. B(c)). One should note that Fig. 5(b) and $\mathrm{B}(\mathrm{c})$ are plotted in the same scale. 


\section{Acknowledgements}

The work was supported as part of Energy Dissipation to Defect Evolution, an Energy Frontier Research Center funded by the U.S. Department of Energy, Office of Science. High-voltage electron irradiation was performed at Ultramicroscopy Research Center at Kyushu University, Japan with technical assistance from Dr. Tomokazu Yamamoto, as a project of the HVEM Collaborative Research Program sponsored by MEXT of the Government of Japan. Instrument support was also provided by Materials Research Science and Engineering Center (DMR-1121288) and Nanoscale Science and Engineering Center (DMR-0832760) at University of Wisconsin-Madison.

* This manuscript has been authored by UT-Battelle, LLC under Contract No. DE-AC05-00OR22725 with the U.S. Department of Energy. The U.S. Government retains and the publisher, by accepting the article for publication, acknowledges that the U.S. Government retains a non-exclusive, paid-up, irrevocable, world-wide license to publish or reproduce the published form of this manuscript, or allow others to do so, for U.S. Government purposes. The Department of Energy will provide public access to these results of federally sponsored research in accordance with the DOE Public Access Plan (http://energy.gov/downloads/doe-public-access-plan).

\section{References}

[1] S.J. Zinkle, J.T. Busby, Structural materials for fission \& fusion energy, Mater. Today 12 (2009) 12-19.

[2] S.J. Zinkle, G.S. Was, Materials challenges in nuclear energy, Acta Mater. 61 (2013) 735-758.

[3] J.W. Yeh, S.K. Chen, S.J. Lin, J.Y. Gan, T.S. Chin, T.T. Shun, C.H. Tsau, S.Y. Chang, Nanostructured high-entropy alloys with multiple principal elements: novel alloy design concepts and outcomes, Adv. Eng. Mater. 6 (2004) 299-303.

[4] B. Cantor, I.T.H. Chang, P. Knight, A.J.B. Vincent, Microstructural development in 
equiatomic multicomponent alloys, Mater. Sci. Eng. A 375-377 (2004) 213-218.

[5] O.N. Senkov, G.B. Wilks, J.M. Scott, D.B. Miracle, Mechanical properties of $\mathrm{Nb}_{25} \mathrm{Mo}_{25} \mathrm{Ta}_{25} \mathrm{~W}_{25}$ and $\mathrm{V}_{20} \mathrm{Nb}_{20} \mathrm{Mo}_{20} \mathrm{Ta}_{20} \mathrm{~W}_{20}$ refractory high entropy alloys, Intermetallics 19 (2011) 698-706.

[6] B. Gludovatz, A. Hohenwarter, D. Catoor, E.H. Chang, E.P. George, R.O. Ritchie, A fracture-resistant high-entropy alloy for cryogenic applications, Science 345 (2014) 1153-1158.

[7] Z.M. Li, K.G. Pradeep, Y. Deng, D. Raabe, C.C. Tasan, Metastable high-entropy dual-phase alloys overcome the strength-ductility trade-off, Nature 534 (2016) 227-230.

[8] Y. Zhang, G.M. Stocks, K. Jin, C. Lu, H. Bei, B.C. Sales, L.M. Wang, L.K. Béland, R.E. Stoller, G.D. Samolyuk, M. Caro, A. Caro, W.J. Weber, Influence of chemical disorder on energy dissipation and defect evolution in concentrated solid solution alloys, Nat. Commun. 6 (2015) 8736.

[9] F. Granberg, K. Nordlund, M.W. Ullah, K. Jin, C. Lu, H. Bei, L.M. Wang, F. Djurabekova, W.J. Weber, Y. Zhang, Mechanism of radiation damage reduction in equiatomic multicomponent single phase alloys, Phys. Rev. Lett. 116 (2016) 135504.

[10] K.C. Russell, Phase stability under irradiation, Prog. Mater. Sci. 28 (1984) 229-434.

[11] A.J. Ardell, P. Bellon, Radiation-induced solute segregation in metallic alloys, Curr. Opin. Solid State Mater. Sci. 20 (2016) 115-139.

[12] Z. Wu, Y. Gao, H. Bei, Thermal activation mechanisms and Labusch-type strengthening analysis for a family of high-entropy and equiatomic solid-solution alloys, Acta Mater. 120 (2016) $108-119$.

[13] M.S. Lucas, G.B. Wilks, L. Mauger, J.A. Muñoz, O.N. Senkov, E. Michel, J. Horwath, S.L. Semiatin, M.B. Stone, D.L. Abernathy, E. Karapetrova, Absence of long-range chemical ordering in equimolar FeCoCrNi, Appl. Phys. Lett. 100 (2012) 257907.

[14] M. Laurent-Brocq, A. Akhatova, L. Perrière, S. Chebini, X. Sauvage, E. Leroy, Y. Champion, Insights into the phase diagram of the CrMnFeCoNi high entropy alloy, Acta Mater. 88 (2015) $355-365$. 
[15] M.S. Lucas, L. Mauger, J.A. Muñoz, Y.M. Xiao, A.O. Sheets, S.L. Semiatin, J. Horwath, Z. Turgut, Magnetic and vibrational properties of high-entropy alloys, J. Appl. Phys. 109 (2011) $07 \mathrm{E} 307$.

[16] B. Schuh, F. Mendez-Martin, B. Völker, E.P. George, H. Clemens, R. Pippan, A. Hohenwarter, Mechanical properties, microstructure and thermal stability of a nanocrystalline CoCrFeMnNi high-entropy alloy after severe plastic deformation, Acta Mater. 96 (2015) 258-268.

[17] F. Otto, A. Dlouhý, K.G. Pradeep, M. Kuběnová, D. Raabe, G. Eggeler, E.P. George, Decomposition of the single-phase high-entropy alloy CrMnFeCoNi after prolonged anneals at intermediate temperatures, Acta Mater. 112 (2016) 40-52.

[18] T. Nagase, P.D. Rack, J.H. Noh, T. Egami, In-situ TEM observation of structural changes in nano-crystalline $\mathrm{CoCrCuFeNi}$ multicomponent high-entropy alloy under fast electron irradiation by high voltage electron microscopy, Intermetallics 59 (2015) 32-42.

[19] N.A.P. Kiran Kumar, C. Li, K.J. Leonard, H. Bei, S.J. Zinkle, Microstructural stability and mechanical behavior of FeNiMnCr high entropy alloy under ion irradiation, Acta Mater. 113 (2016) 230-244.

[20] X.M. Bai, A.F. Voter, R.G. Hoagland, M. Nastasi, B.P. Uberuaga, Efficient annealing of radiation damage near grain boundaries via interstitial emission, Science 327 (2010) 1631-1634. [21] A. Etienne, B. Radiguet, N.J. Cunningham, G.R. Odette, R. Valiev, P. Pareige, Comparison of radiation-induced segregation in ultrafine-grained and conventional 316 austenitic stainless steels, Ultramicroscopy 111 (2011) 659-663.

[22] Z. Wu, Y. Gao, H. Bei, Single crystal plastic behavior of a single-phase, face-center-cubic-structured, equiatomic FeNiCrCo alloy, Scripta Mater. 109 (2015) 108-112.

[23] F. Otto, Y. Yang, H. Bei, E.P. George, Relative effects of enthalpy and entropy on the phase stability of equiatomic high-entropy alloys, Acta Mater. 61 (2013) 2628-2638.

[24] M.R. He, S. Wang, K. Jin, H. Bei, K. Yasuda, S. Matsumura, K. Higashida, I.M. Robertson, Enhanced damage resistance and novel defect structure of $\mathrm{CrFeCoNi}$ under in situ electron 
irradiation, Scripta Mater. 125 (2016) 5-9.

[25] P. Czurratis, R. Kroggel, V. Syneček, M. Simerska, H. Löffler, Dislocation loops in Al-Zn and Al-Zn-Mg alloys, Phys. Stat. Sol. A 106 (1988) 407-418.

[26] G. Thomas, J. Washburn, Precipitation of vacancies in metals, Rev. Mod. Phys. 35 (1963) 992-1011.

[27] J.W. Yeh, Physical metallurgy of high-entropy alloys, JOM 67 (2015) 2254-2261.

[28] T. Kato, H. Takahashi, M. Izumiya, Grain boundary segregation under electron irradiation in austenitic stainless steels modified with oversized elements, J. Nucl. Mater. 189 (1992) 167-174. [29] D.S. Aidhy, C. Lu, K. Jin, H. Bei, Y. Zhang, L.M. Wang, W.J. Weber, Point defect evolution in $\mathrm{Ni}, \mathrm{NiFe}$ and $\mathrm{NiCr}$ alloys from atomistic simulations and irradiation experiments, Acta Mater. 99 (2015) 69-76.

[30] Y.N. Osetsky, L.K. Béland, R.E. Stoller, Specific features of defect and mass transport in concentrated fcc alloys, Acta Mater. 115 (2016) 364-371.

[31] H. Wiedersich, P.R. Okamoto, N.Q. Lam, A theory of radiation-induced segregation in concentrated alloys, J. Nucl. Mater. 83 (1979) 98-108.

[32] A. Takeuchi, A. Inoue, Classification of bulk metallic glasses by atomic size difference, heat of mixing and period of constituent elements and its application to characterization of the main alloying element, Mater. Trans. 46 (2005) 2817-2829.

[33] J. Teeriniemi, P. Taskinen, and K. Laasonen, First-principles investigation of the Cu-Ni, Cu-Pd, and Ni-Pd binary alloy systems, Intermetallics 57 (2015) 41-50.

[34] J.M. Zuo, J.C. Mabon, WebEMAPS, http://emaps.mrl.uiuc.edu/emaps.asp.

[35] A.R. Denton, N.W. Ashcroft, Vegard's law, Phys. Rev. A 43 (1991) 3161-3164.

[36] J.M. Cowley, Diffraction Physics, North-Holland, Amsterdam, 1975.

[37] R. Oshima, M. Sugiyama, F.E. Fujita, Tweed structures associated with fcc-fct transformations in Fe-Pd alloys, Metall. Trans. A 19 (1988) 803-810.

[38] Y. Le Bouar, A. Loiseau, A.G. Khachaturyan, Origin of chessboard-like structures in decomposing alloys. Theoretical model and computer simulation, Acta Mater. 46 (1998) 
$2777-2788$.

[39] I.M. Robertson, C.M. Wayman, Tweed microstructures I. Characterization in $\beta$-NiAl, Phil. Mag. A 48 (1983) 421-442.

[40] G. Simmons, H. Wang, Single Crystal Elastic Constants and Calculated Aggregate Properties: A Handbook, MIT Press, Cambridge, 1971.

[41] J.W. Cahn, On spinodal decomposition in cubic crystals, Acta Metall. 10 (1962) 179-183.

[42] G. Ghosh, C. Kantner, G.B. Olson, Thermodynamic modeling of the Pd-X (X = Ag, Co, Fe, Ni) systems, J. Phase Equilib. 20 (1999) 295-308.

[43] W. Lin, J.E. Spruiell, The structure of nickel-palladium solid solutions, Acta Metall. 19 (1971) 451-461.

[44] R. Cauvin, G. Martin, Solid solutions under irradiation II. Radiation-induced precipitation in AlZn undersaturated solid solutions, Phys. Rev. B 23 (1981) 3333-3348.

[45] Y. Zhang, Y.J. Zhou, J.P. Lin, G.L. Chen, P.K. Liaw, Solid-solution phase formation rules for multi-component alloys, Adv. Eng. Mater. 10 (2008) 534-538.

[46] S. Guo, Q. Hu, C. Ng, C.T. Liu, More than entropy in high-entropy alloys: Forming solid solutions or amorphous phase, Intermetallics 41 (2013) 96-103.

[47] S.J. Zinkle, P.J. Maziasz, R.E. Stoller, Dose dependence of the microstructural evolution in neutron-irradiated austenitic stainless steel, J. Nucl. Mater. 206 (1993) 266-286.

[48] T.R. Allen, J.T. Busby, G.S. Was, E.A. Kenik, On the mechanism of radiation-induced segregation in austenitic Fe-Cr-Ni alloys, J. Nucl. Mater. 255 (1998) 44-58.

[49] A. Etienne, B. Radiguet, P. Pareige, Understanding silicon-rich phase precipitation under irradiation in austenitic stainless steels, J. Nucl. Mater. 406 (2010) 251-256.

[50] G.S. Was, Fundamentals of Radiation Materials Science, Springer, Berlin, 2007.

[51] C. Lu, et al., unpublished results.

[52] E.A. Kenik, Radiation-induced segregation in irradiated Type 304 stainless steels, J. Nucl. Mater. 187 (1992) 239-246.

[53] S. Watanabe, Y. Takamatsu, N. Sakaguchi, H. Takahashi, Sink effect of grain boundary on 
radiation-induced segregation in austenitic stainless steel, J. Nucl. Mater. 283-287 (2000) $152-156$.

[54] Z. Jiao, G.S. Was, Novel features of radiation-induced segregation and radiation-induced precipitation in austenitic stainless steels, Acta Mater. 59 (2011) 1220-1238.

[55] L.E. Rehn, P.R. Okamoto, H. Wiedersich, Dose dependence of radiation-induced segregation in Ni-1 at\% Si, J. Nucl. Mater. 80 (1979) 172-179.

[56] W. Wagner, L.E. Rehn, H. Wiedersich, V. Naundorf, Radiation-induced segregation in Ni-Cu alloys, Phys. Rev. B 28 (1983) 6780-6794.

[57] M.J. Berger, J.S. Coursey, M.A. Zucker, J. Chang, Stopping-Power \& Range Tables for Electrons, http://physics.nist.gov/PhysRefData/Star/Text/ESTAR.html.

[58] K. Jin, B.C. Sales, G.M. Stocks, G.D. Samolyuk, M. Daene, W.J. Weber, Y. Zhang, H. Bei, Tailoring the physical properties of Ni-based single-phase equiatomic alloys by modifying the chemical complexity, Sci. Rep. 6 (2016) 20159. 

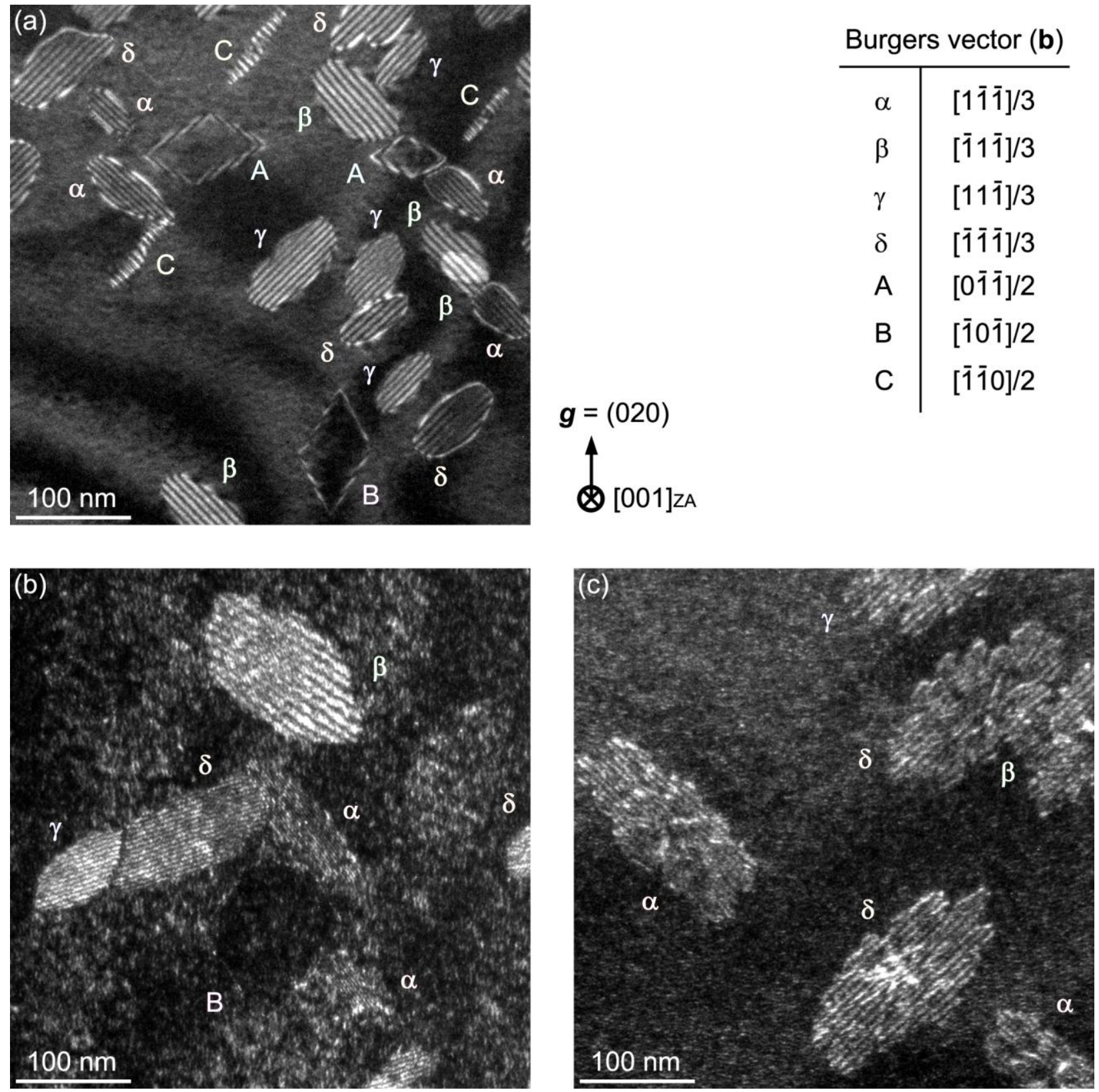

Fig. 1. Representative defect structure in (a) $\mathrm{CrFeCoNi}$, (b) $\mathrm{CrFeCoNiMn}$, and (c) $\mathrm{CrFeCoNiPd}$ as irradiated by $1250 \mathrm{kV}$ electrons at $400{ }^{\circ} \mathrm{C}$ up to $1 \mathrm{dpa}$, all viewed in (g, 3.1 g) WBDF condition with $\mathbf{g}=(020)$ near [001] ZA. The symbol of each dislocation loop denotes its Burgers vector. 

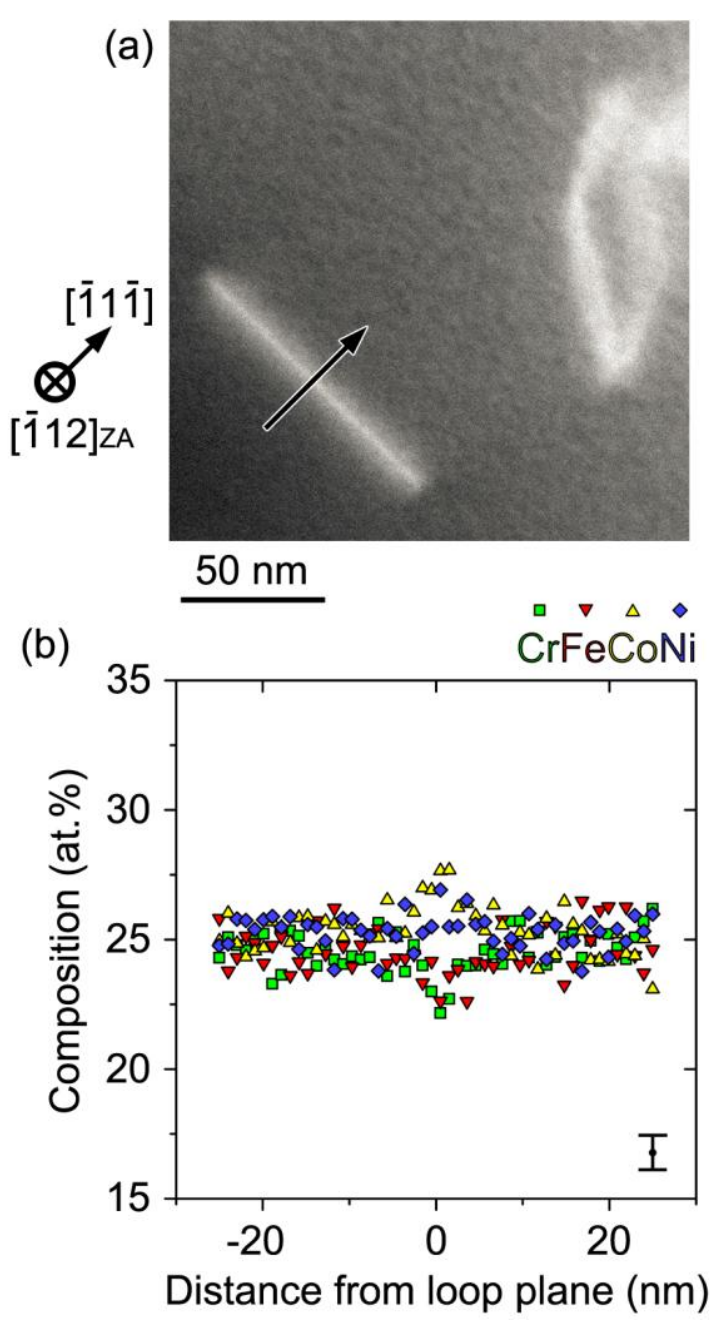
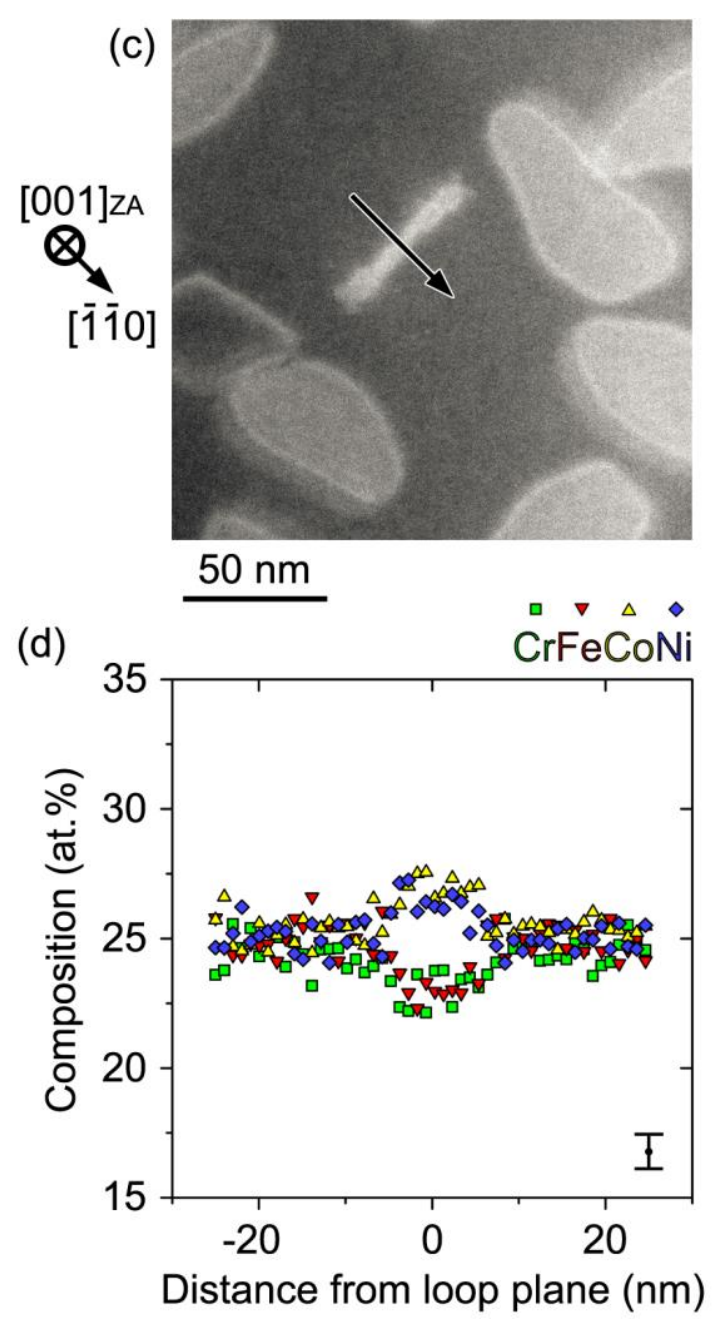

Fig. 2. RIS at (a,b) Frank loop and (c,d) perfect loop in CrFeCoNi. (a,c) ADF-STEM edge-on views of the loops. (b,d) EDS line-scans across the habit planes, i.e., along arrows in (a,c). Error bar shows the standard deviation of composition at each measurement point. 

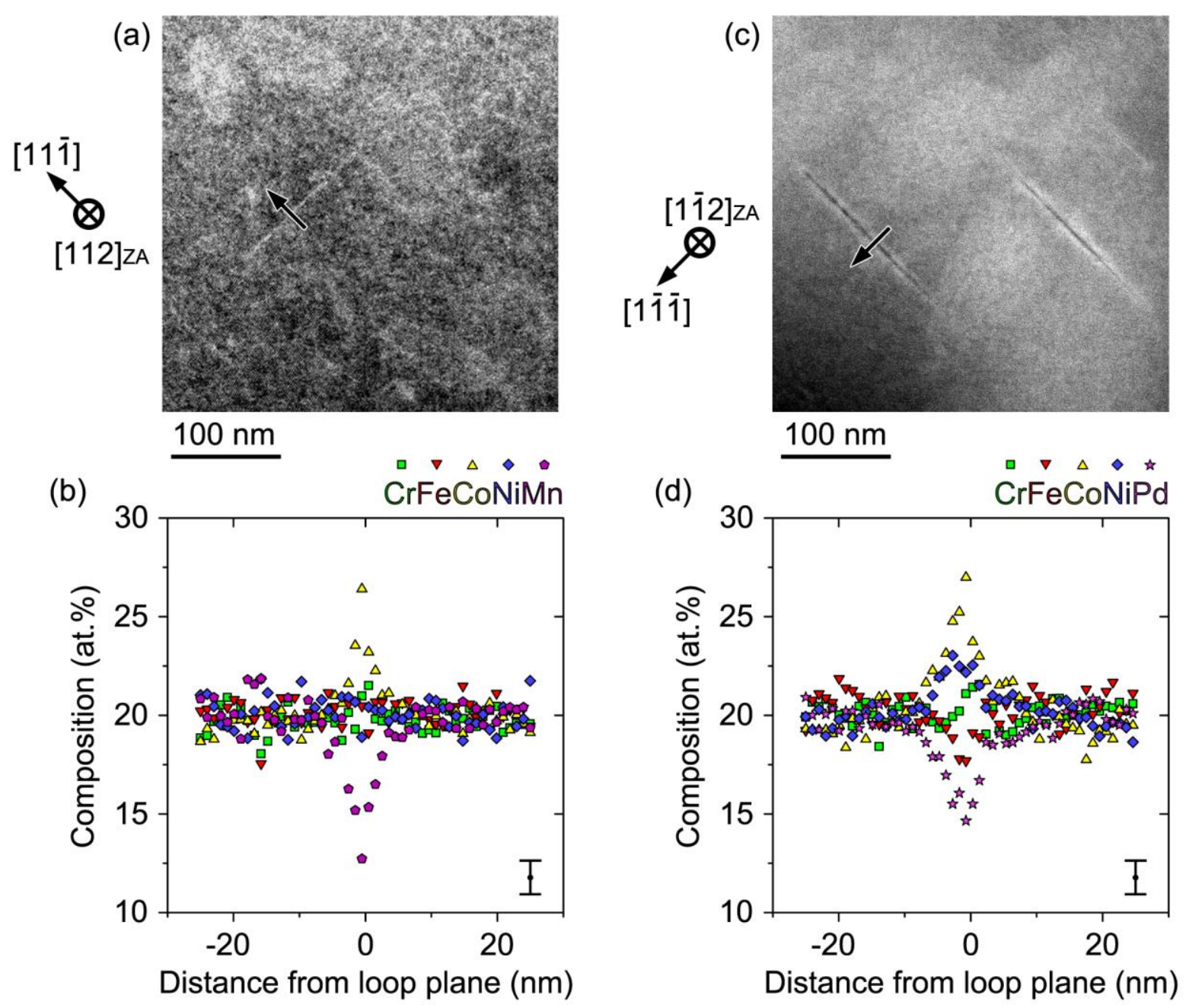

Fig. 3. RIS at Frank loops in (a,b) CrFeCoNiMn and (c,d) CrFeCoNiPd. (a,c) ADF-STEM edge-on views of the loops. (b,d) EDS line-scans across the habit planes, i.e., along arrows in $(\mathrm{a}, \mathrm{c})$. 


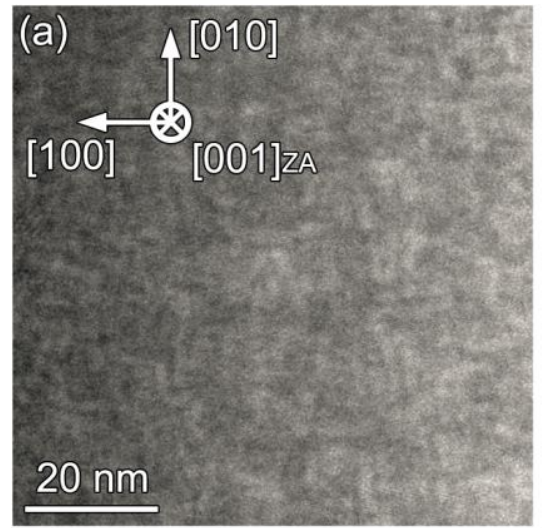

(b)

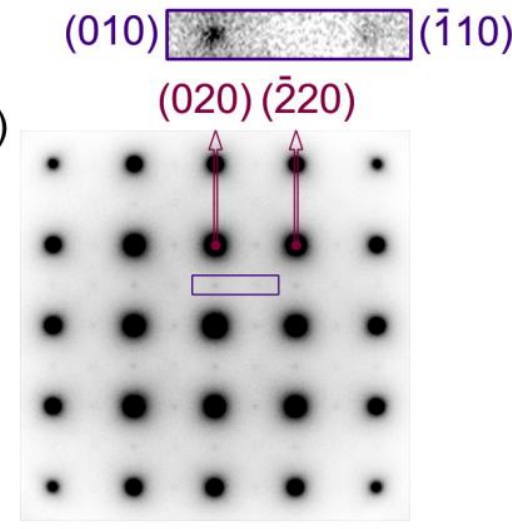

(d)
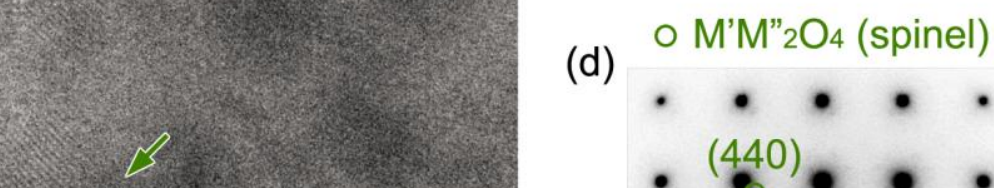

(f)

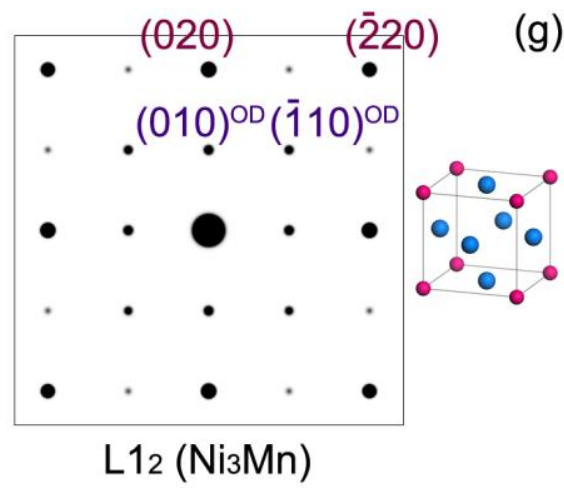

(g)

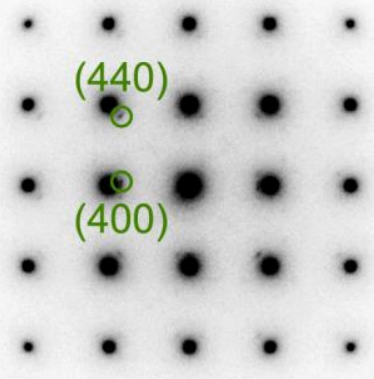

\section{$20 \mathrm{~nm}$}

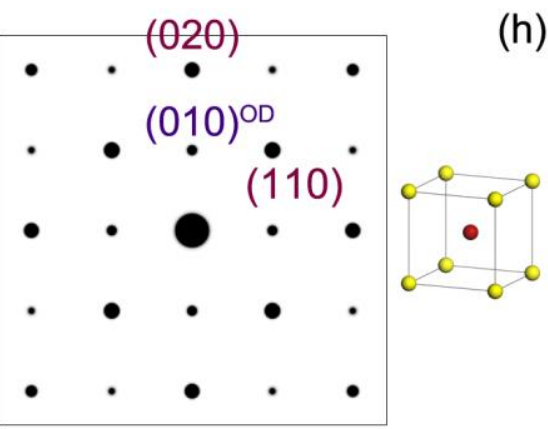

$\mathrm{B} 2(\mathrm{FeCo})$ (e)

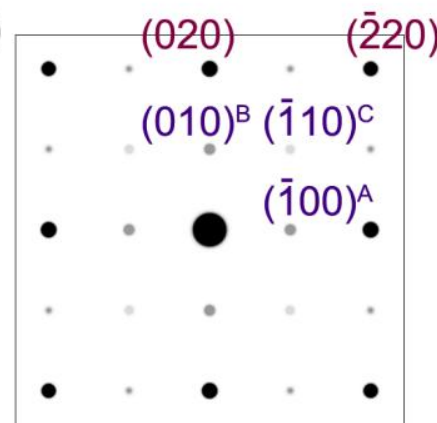

$40 \%$ (A) $+40 \%$ (B) $+20 \%$ (C)

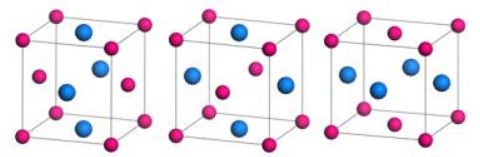

L10 (NiMn)

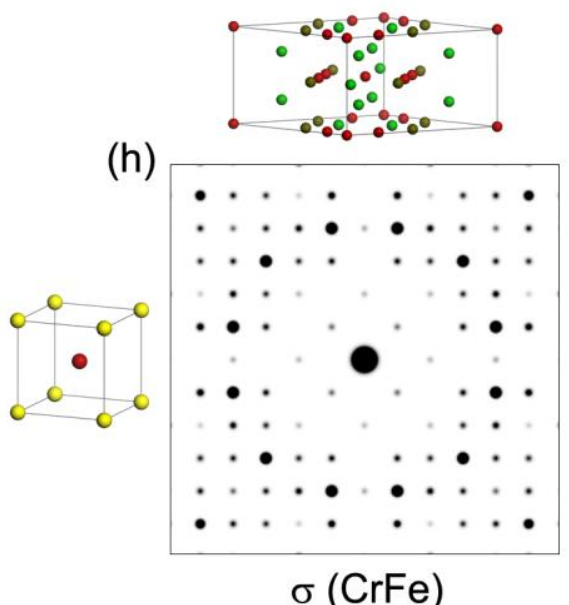

$\sigma(\mathrm{CrFe})$

Fig. 4. Ordering in electron-irradiated matrix of CrFeCoNiMn. (a) ADF-STEM image and (b) corresponding SADP in [001] ZA. Enlarged view of box highlights $\{100\}$ and $\{110\}$ families of extra spots. (c) ADF-STEM image and (d) SADP of un-irradiated matrix in [001] ZA. Arrows in (c) and circles in (d) correspond to spinel-type surface oxides that are near-epitaxial to FCC matrix. (e) Simulated SADP yielded by a mixture of $\mathrm{L}_{1}$-type ordered NiMn phase in all three orientations. (f-h) Simulated SADPs of other types of ordered (OD) phases with cubic/tetragonal structures: (f) $\mathrm{L1}_{2}-\mathrm{Ni}_{3} \mathrm{Mn}$; (g) B2-FeCo; (h) $\sigma-\mathrm{Cr}_{1-x} \mathrm{Fe}_{1+x}$. All SADPs are in [001] ZA. 
(a)
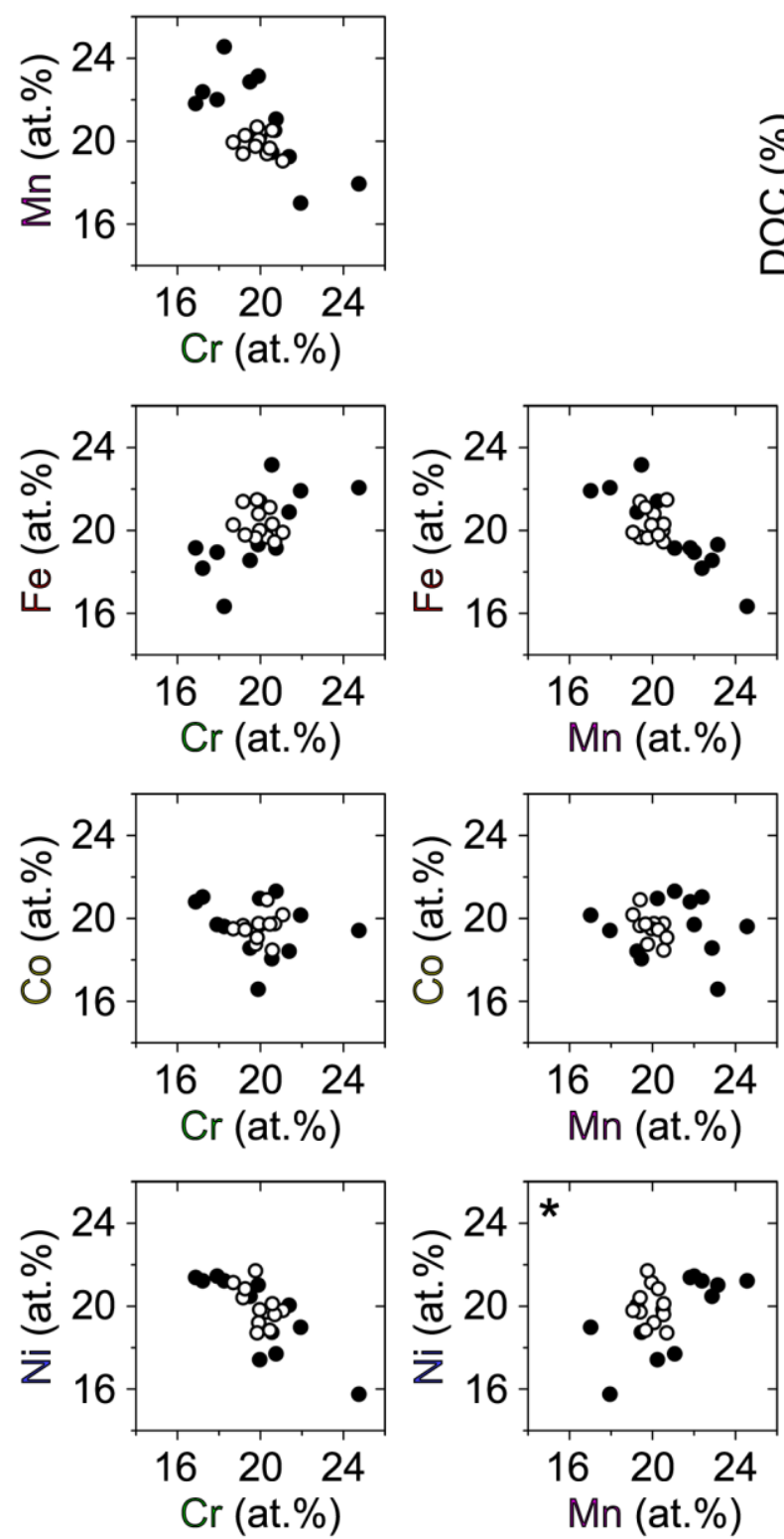

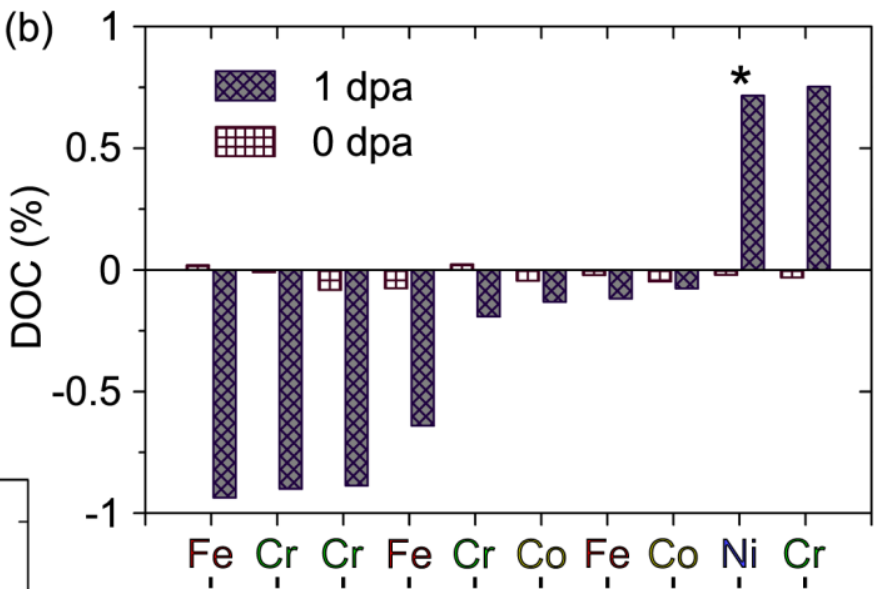

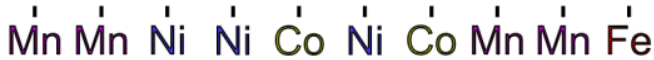
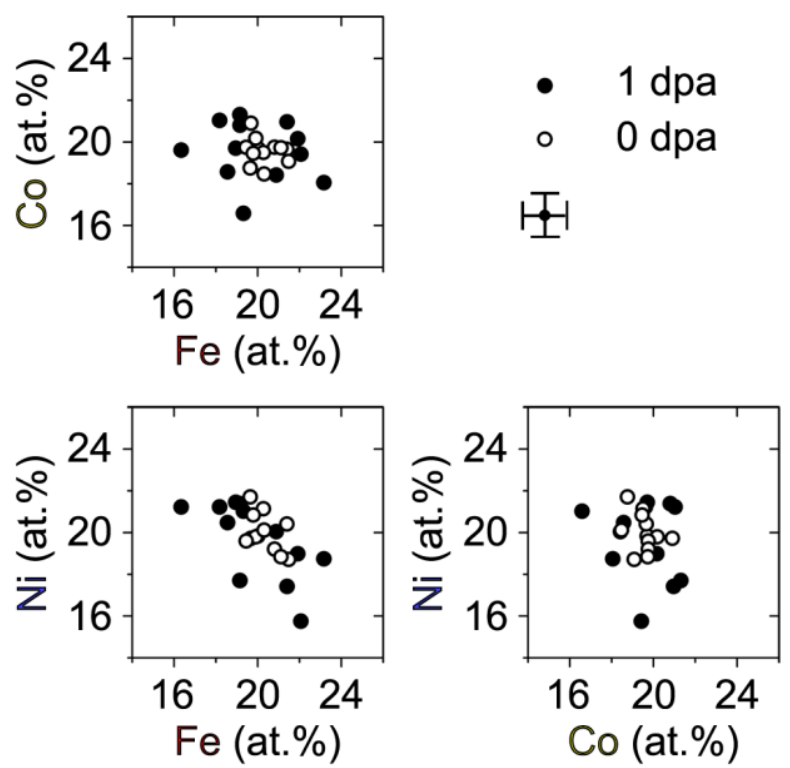

Fig. 5. Decomposition in electron-irradiated matrix of CrFeCoNiMn. (a) Correlation map of all element pairs in un-irradiated (open circles) and irradiated (solid circles) matrix as measured by EDS. (b) DOC of all element pairs. Concentrations of Ni-Mn (*) show a significantly augmented and positive covariance due to irradiation. 


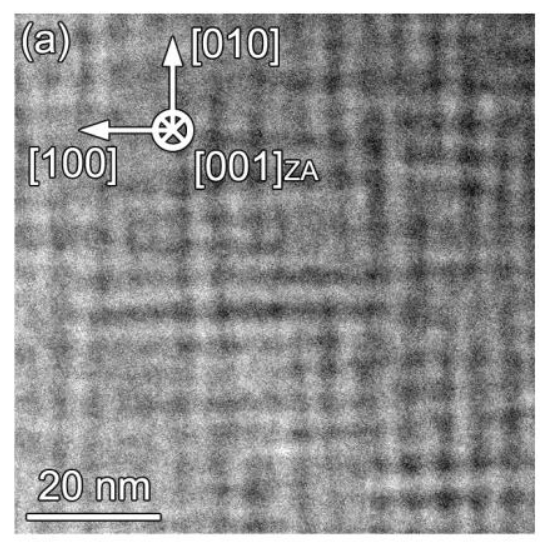

(b)
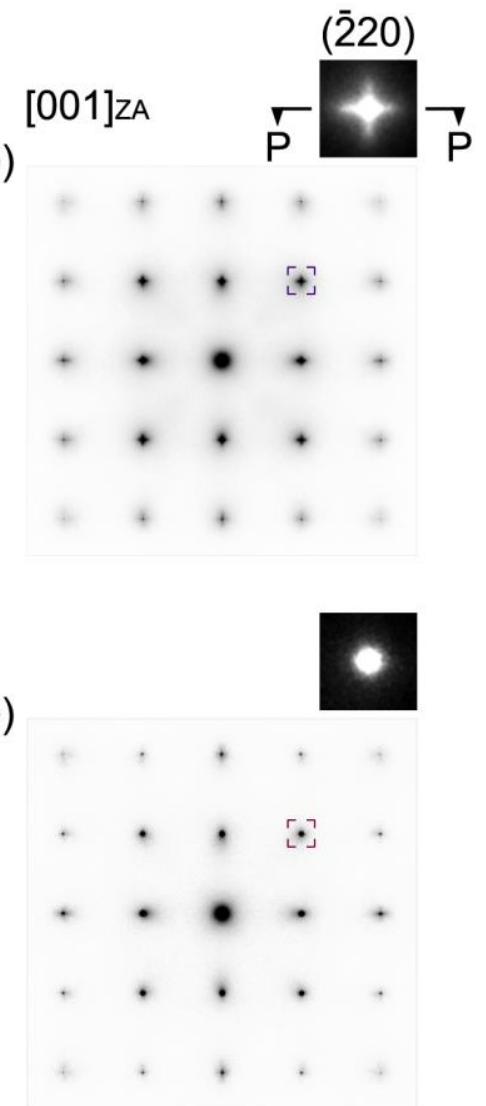

(e) (c)

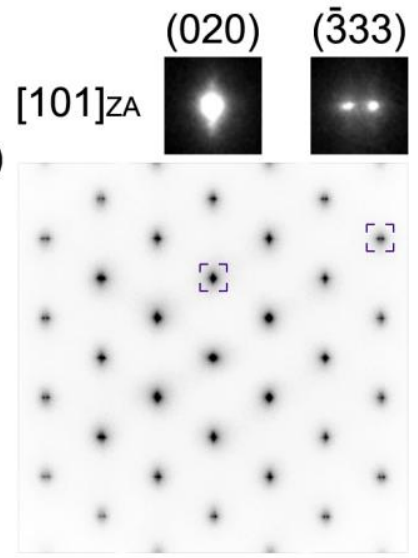

(f)
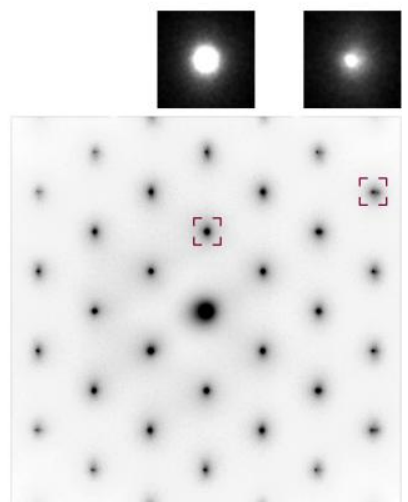

(g)

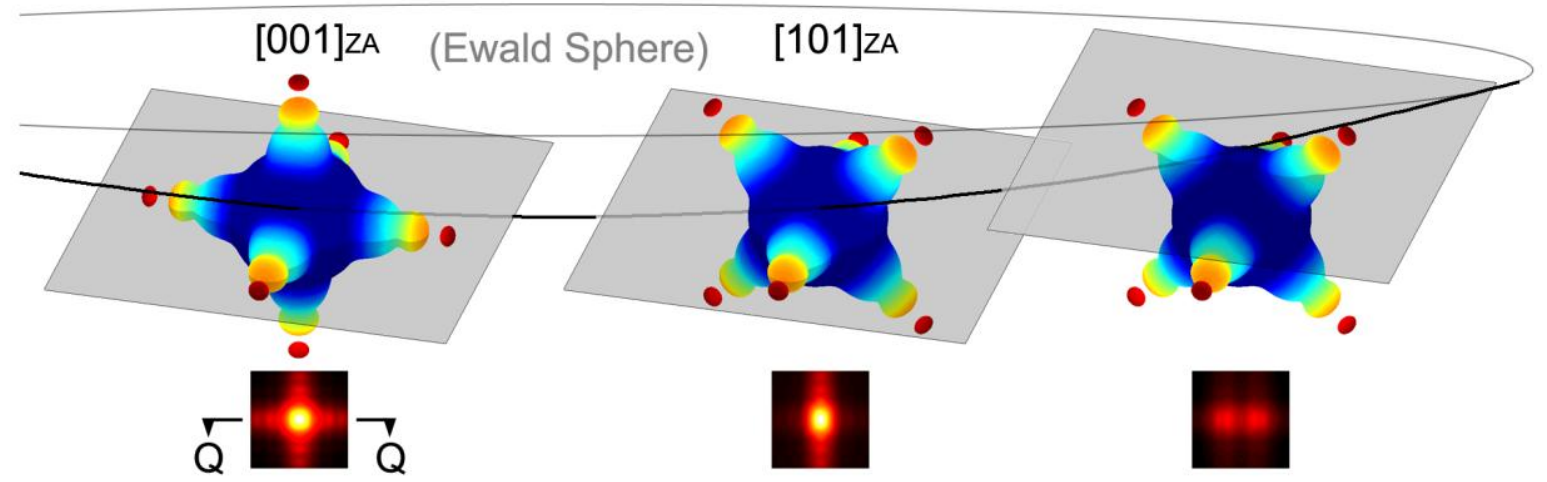

Fig. 6. Modulated structure in electron-irradiated matrix of CrFeCoNiPd. (a) ADF-STEM image and corresponding SADPs in (b) [001] and (c) [101] ZAs, respectively. Enlarged views of boxes highlight the streaks and splits of diffraction spots. (d) ADF-STEM image and (e,f) SADPs of featureless, un-irradiated matrix. (g) Schematic of $<001>$-oriented streaking in three-dimensional reciprocal space. Diffraction are calculated by intersecting reciprocal sites using Ewald sphere. 
(a)
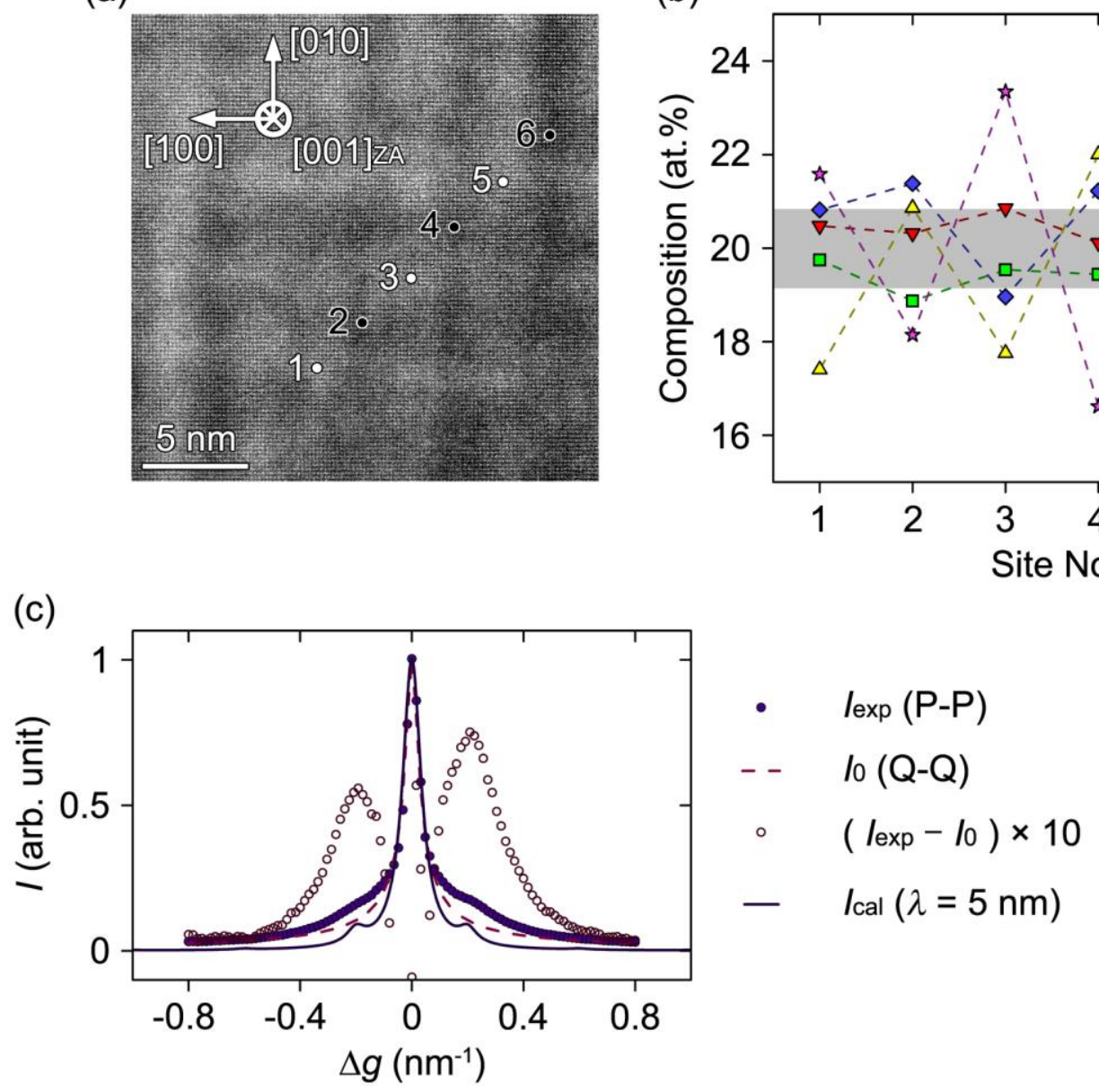

(b)
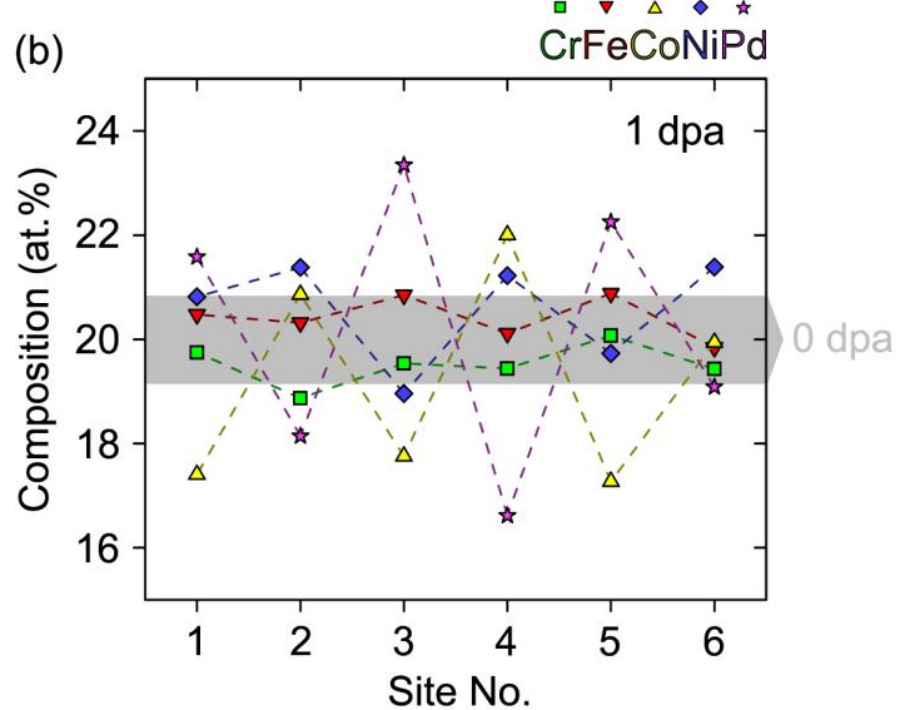

Fig. 7. Spinodal decomposition in electron-irradiated matrix of $\mathrm{CrFeCoNiPd}$. (a) Enlarged ADF-STEM image shows no extra phase. (b) EDS measurements at alternating locations with bright and dark contrasts in (a). Shadow shows the compositional range of all elements in un-irradiated matrix. (c) Solid circles: intensity profile of $\{220\}$ reflections along [100] direction (P-P) in Fig. 6(b). Dash line: intensity of central peak fitted by Lorentz distribution. Open circles highlight the diffuse intensity. Solid line: calculated diffraction profile (e.g., along Q-Q in Fig. $6(\mathrm{~g}))$ of a one-dimensional sinusoidal wave with a wavelength $(\lambda)=5 \mathrm{~nm}$. 

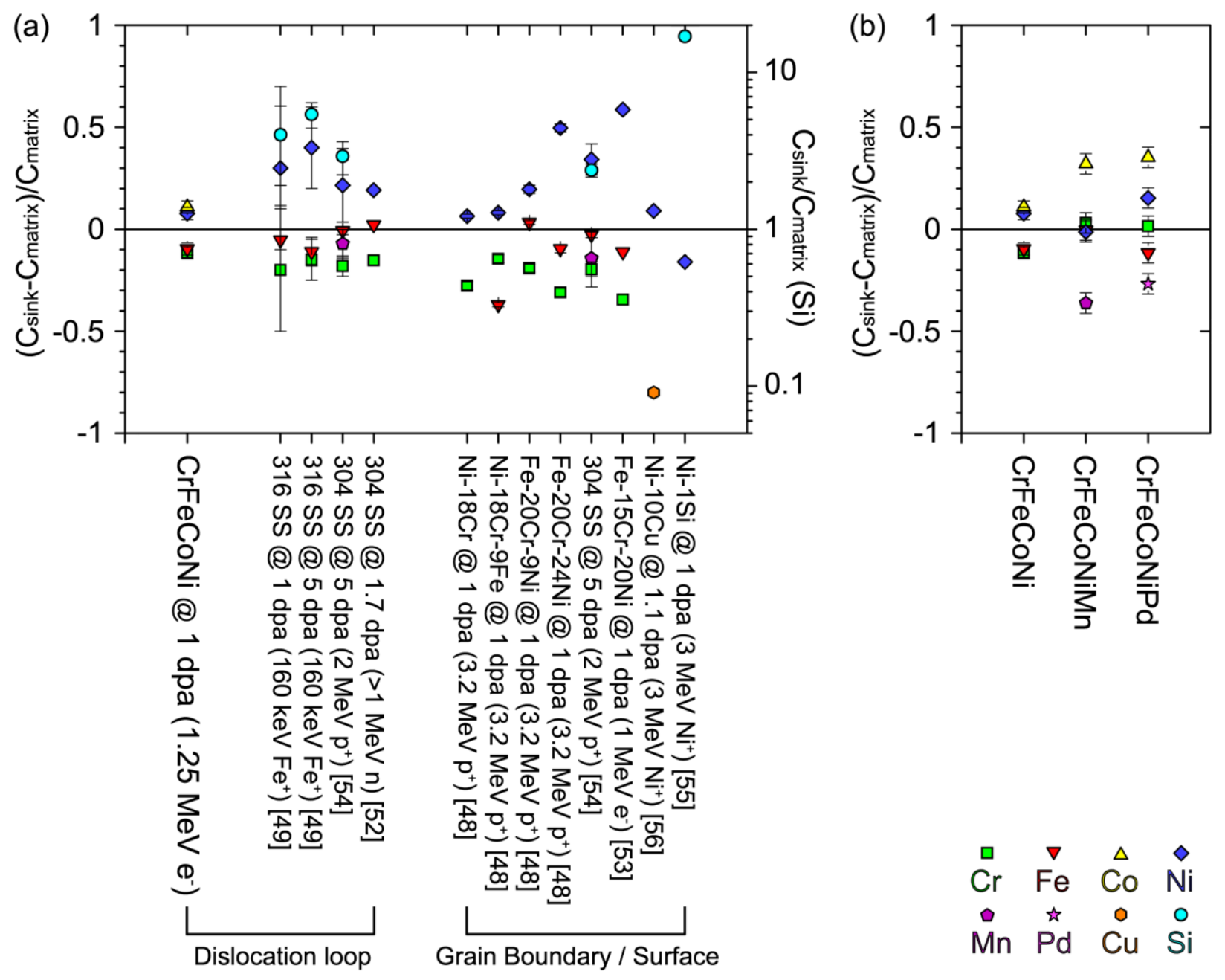

Fig. 8. Magnitude of RIS, i.e., change of composition relative to that of un-irradiated matrix, at defect sinks. (a) Comparison between $\mathrm{CrFeCoNi}$ (in this study) and common austenitic stainless steels and Ni-based binary alloys as irradiated to similar damage levels and at temperatures around $400{ }^{\circ} \mathrm{C}$. (b) Comparison between the three alloys in this study.

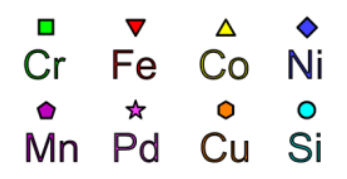




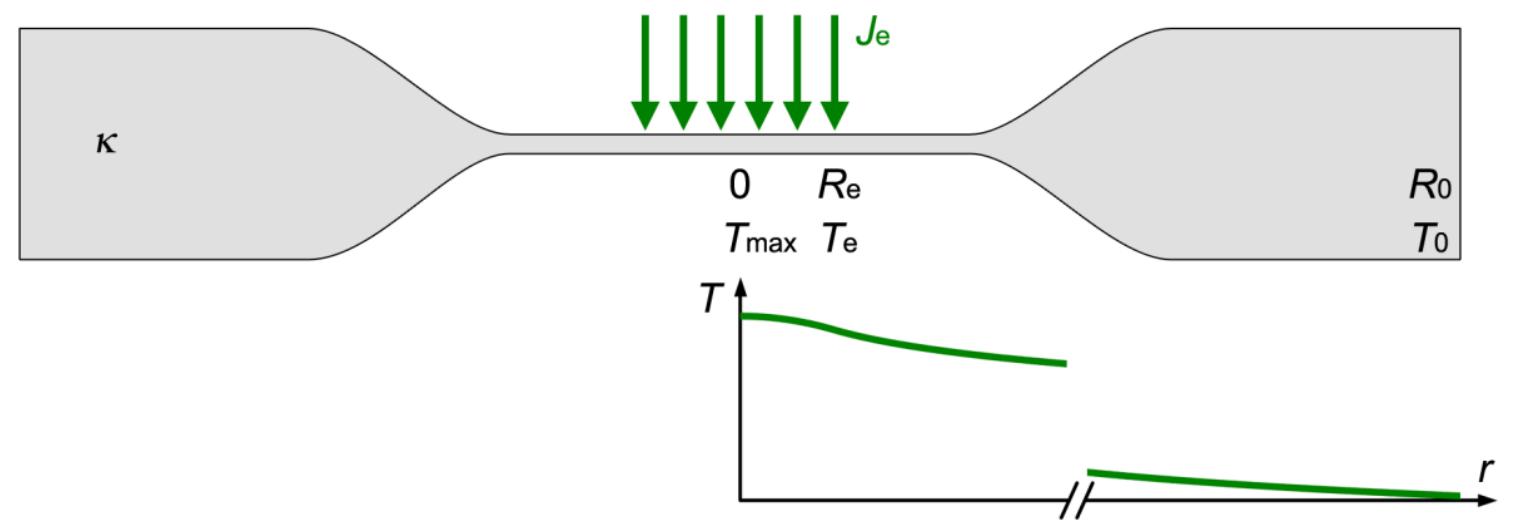

Fig. A. Schematic of a TEM thin foil (not plotted to scale) under electron irradiation and the calculation of steady temperature increase.

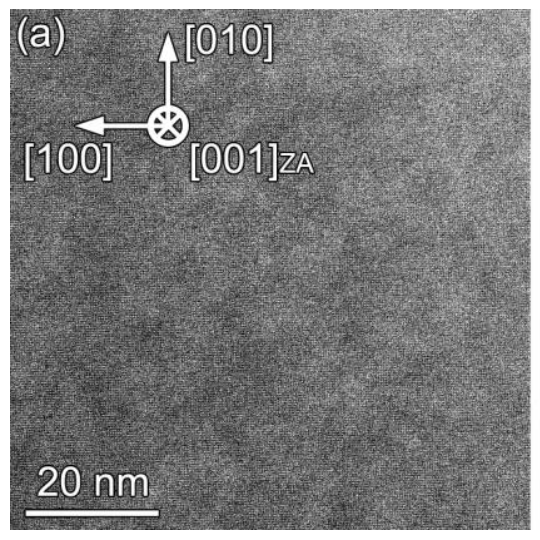

(b)

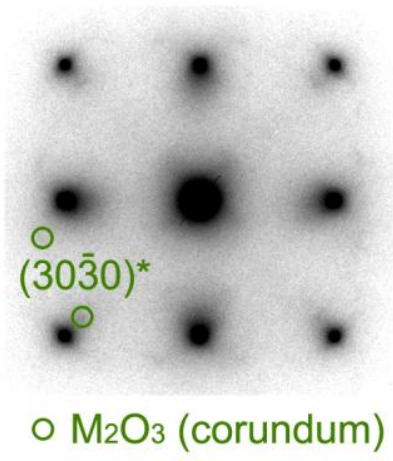

(c) 曲 1 dpa

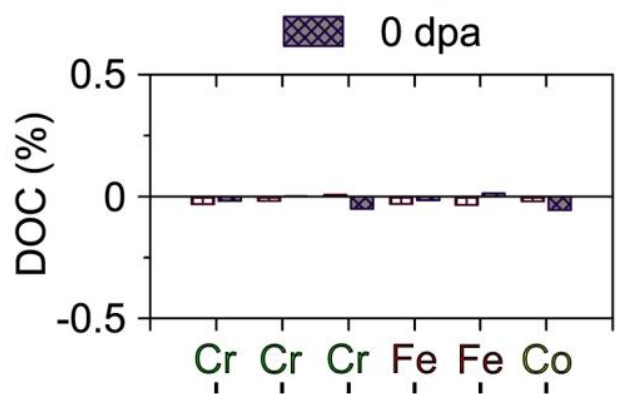

Fe ćo Ni Ćo Ni Ni

Fig. B. Electron-irradiated matrix of CrFeCoNi. (a) ADF-STEM image and (b) corresponding SADP in [001] ZA. (c) DOC of all element pairs in the un-irradiated and irradiated matrix. 
Table 1. Atomic radius $(a)$ of all relevant elements (metallic, coordinate number $=12$ ) and enthalpy of mixing $\left(\Delta H_{\text {mix }}\right)$ between each pair of elements. Data are obtained from [32], $\dagger$ $[23]$, and (§) [33].

\begin{tabular}{|c|c|c|c|c|c|c|}
\hline & $\mathrm{Cr}$ & $\mathrm{Fe}$ & $\mathrm{Co}$ & $\mathrm{Ni}$ & $\mathrm{Mn}$ & $\mathrm{Pd}$ \\
\hline $\begin{array}{c}a \\
(\mathrm{pm})\end{array}$ & 127.1 & 128.2 & 125.3 & 124.6 & 130.7 & 137.5 \\
\hline $\begin{array}{c}\Delta H_{\text {mix }} \\
\left(\mathrm{kJ} \cdot \mathrm{mol}^{-1}\right)\end{array}$ & & & & & & \\
\hline $\mathrm{Cr}$ & & -1 & -4 & $-7 /-2^{\dagger}$ & $+2 /-3^{\dagger}$ & -15 \\
\hline $\mathrm{Fe}$ & & & -1 & -2 & 0 & -4 \\
\hline Co & & & & 0 & -5 & -1 \\
\hline $\mathrm{Ni}$ & & & & & $-8 /-11^{\dagger}$ & $0 /+4^{\S}$ \\
\hline
\end{tabular}



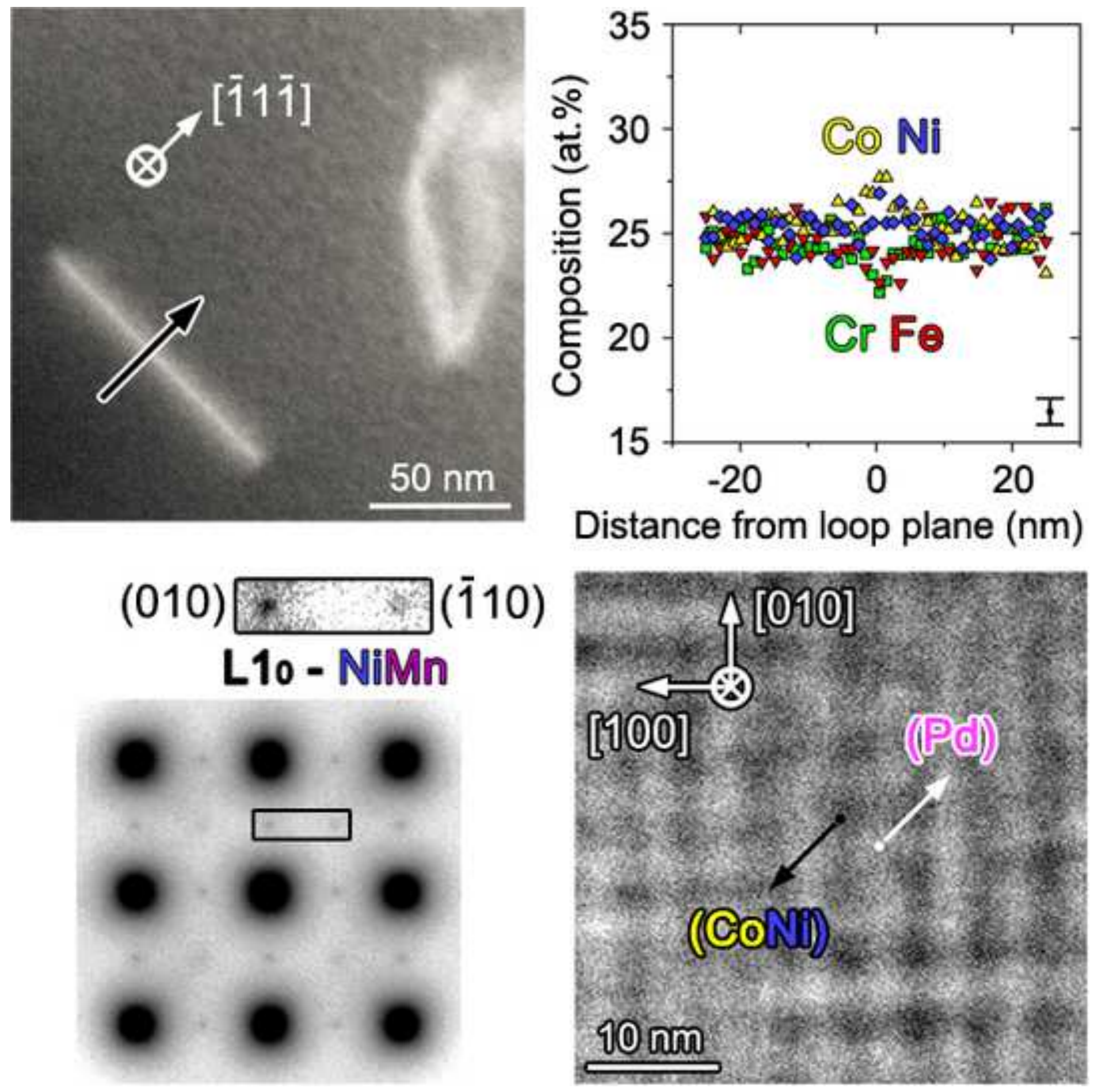

$50 \mathrm{~nm}$

$$
\begin{aligned}
& \text { (G) } \\
& m
\end{aligned}
$$

